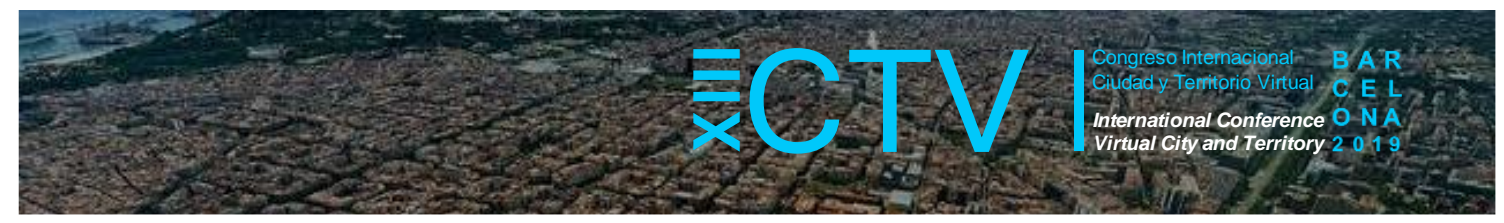

\title{
GIRONA SISTEMA URBANO: ANÁLISIS DEL CLIMA URBANO DE UN SISTEMA DE CIUDADES MEDIAS CATALANAS USANDO REMOTE SENSING
}

\author{
Zambrano Loor, Fernando Javier ${ }^{1 *}$; Rodríguez Antuñano, Ignacio ${ }^{2}$ y Durango Cordero, \\ Santiago $^{3}$
}

Remisión inicial: 2019-06-16; Remisión definitiva: 2019-10-28; Publicación: 2019-12-21
Citación: Zambrano, F. et al. (2019). Girona sistema urbano: análisis del clima urbano de un sistema de ciudades medias catalanas usando Remote Sensing. En XIII CTV 2019 Proceedings: XIII International Conference on Virtual City and Territory: "Challenges and paradigms of the contemporary city”: UPC, Barcelona, October 2-4, 2019. Barcelona: CPSV, 2019, p. 8623. E-ISSN 2604-6512. DOI http://dx.doi.org/10.5821/ctv.8623

\begin{abstract}
Resumen
Hasta el momento, el diseño urbano no tomaba en cuenta factores medioambientales más allá de cuestiones estéticas. Sin embargo, con las constantes evidencias del cambio climático cada vez es más importante que la planificación urbana se realice en base a varios indicadores climáticos. Los climatopes son una herramienta básica para el futuro diseño de las ciudades, así como para tomar decisiones para enfrentarse al calentamiento global por parte de los urbanistas y políticos. La idea de climatope consiste en que las diferentes estructuras urbanas interactúen de manera distinta entre sí, pudiendo agruparse por características comunes, consiguiendo por ello identificar áreas sensibles donde existan problemas urbanos y ambientales.
\end{abstract}

La idea de climatope surge en Alemania en la década de 1970, adaptándose a la mayoría de las ciudades alemanas, así como al resto de ciudades europeas. Sin embargo, en España solo se ha hecho estudios en contadas ciudades importantes, es por ello que se hace especialmente necesario hacer el estudio de climatopes en ciudades medias. Por ciudad media entendemos un sistema urbano, es decir, no solo una ciudad si no también los municipios que configuran su entorno urbano. Para ello se usan una serie de indicadores, tales como NDVI, NDBI, LST, EBBI, IBBI y DMT obtenidos mediante sistemas de información geográfica. El presente estudio pretende, mediante el análisis de clima urbano y remote sensing, determinar la existencia de isla de calor urbano de una ciudad media de Cataluña, que no forme parte del área metropolitana de Barcelona, siendo Girona la elegida para el estudio debido a su importancia dentro de Cataluña.

\section{Abstract}

Urban design did not take into account environmental factors beyond aesthetic issues. However, with the constant evidence of climate change, it is increasingly important that urban planning be carried out based on various climate indicators. Climatopes are a basic tool for the future design of cities, as well as to make decisions to face global warming by urban planners and politicians. The idea of climatope is that different urban structures interact differently with each other, and can be grouped by common characteristics, thereby identifying sensitive areas where there are urban and environmental problems.

The idea of climatope arises in Germany in the 1970s, adapting to most German cities, as well as to other European cities. However, in Spain only studies have been done in a few important cities, which is why it is especially necessary to study climatopes in medium cities. By average city we understand an urban system, that is, not only a city but also the municipalities that make up its urban environment. For this, a series of indicators are used, such as NDVI, NDBI, LST, EBBI, IBBI and DMT obtained through geographic information systems. The present study intends, through the analysis of urban climate and remote sensing, to determine the existence of an urban heat island of an average city in Catalonia, which is not part of the metropolitan area of Barcelona, being Girona the one chosen for the study due to its importance within Catalonia.

Palabras Clave: Remote Sensing; Sistema Urbano; Climatopes; Planeamiento urbano

Key words: Remote Sensing; Urban System; Climatopes; Urban Planning

\footnotetext{
1 MBArch-UPC, línea de Gestión y Valoración Urbana y Arquitectónica, https://orcid.org/0000-0003-3209-7452; ${ }^{2}$ MBArch-UPC, línea de Gestión y Valoración Urbana y Arquitectónica, https://orcid.org/0000-0001-7784-0540; y ${ }^{3}$ MBArch-UPC, línea de Gestión y Valoración Urbana y Arquitectónica, https://orcid.org/0000-0002-9967-1095. *Correo
} de contacto: inandofer1@hotmail.com 


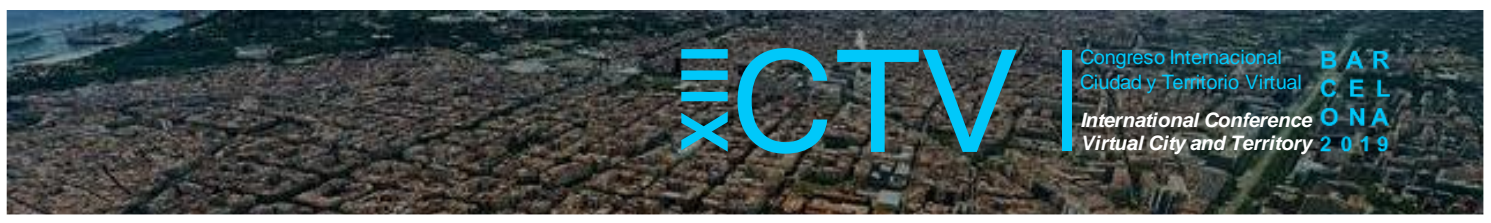

\section{Definición del Sistema Urbano}

Para decidir la elección del sistema urbano de Girona partimos desde la definición de "ciudad media" por el Banco Mundial, la cual considera que la categoría de ciudad media debe tener hasta 1.000.000 de habitantes, y para la Unión Europea el espectro se mueve entre los 50.000 y 500.000 habitantes (Bellet \& Llop, 2001).

Existe ya una definición del sistema urbano de Girona y lo hace el Plan Territorial General de Cataluña el cual lo define como una entidad de población que aglomera distintos municipios, con un elevado potencial de crecimiento demográfico y de actividad, y que puede ser capaz de competir con el sistema central metropolitano de Barcelona. Fundamentalmente se trata de los municipios de Gerona, Aiguaviva, Bescanó, Celrá, Fornells de la Selva, Llambillas, Quart, Riudellots de la Selva, Salt, San Gregorio, San Julian de Ramis, Sarriá de Ter, Vilablareix y Viloví de Oñar.

\subsection{Estudios Previos}

\subsubsection{Ensayo de Definición del Área Urbana de Girona}

Para determinar áreas metropolitanas se suele basar en relaciones residencia-trabajo. También llamadas funcionales. En el ensayo, realizado por Castañer, Vicente, Comas y Box por la universidad de Girona, se define el sistema urbano de Girona con los datos de 1986 y 1991 mediante la superposición y análisis de los distintos modelos de territorio que tienen como variable la movilidad laboral. Ellos terminan diferenciando en dos épocas distintas (1986 y 1991) el sistema urbano de Girona por medio de estas relaciones, en la cual le asignan tres categorías o coronas al sistema urbano para determinar sus áreas de influencia y de crecimiento. Así para el año 1991 se mantienen 22 municipios, pero existen ya dos municipios más que pertenecen a la primera corona de ciudades, estas son San Gregori y Sant Julia de Ramis.

Figura 1. Niveles de áreas de influencia de Girona (1986). Delimitación a partir de la movilidad laboral

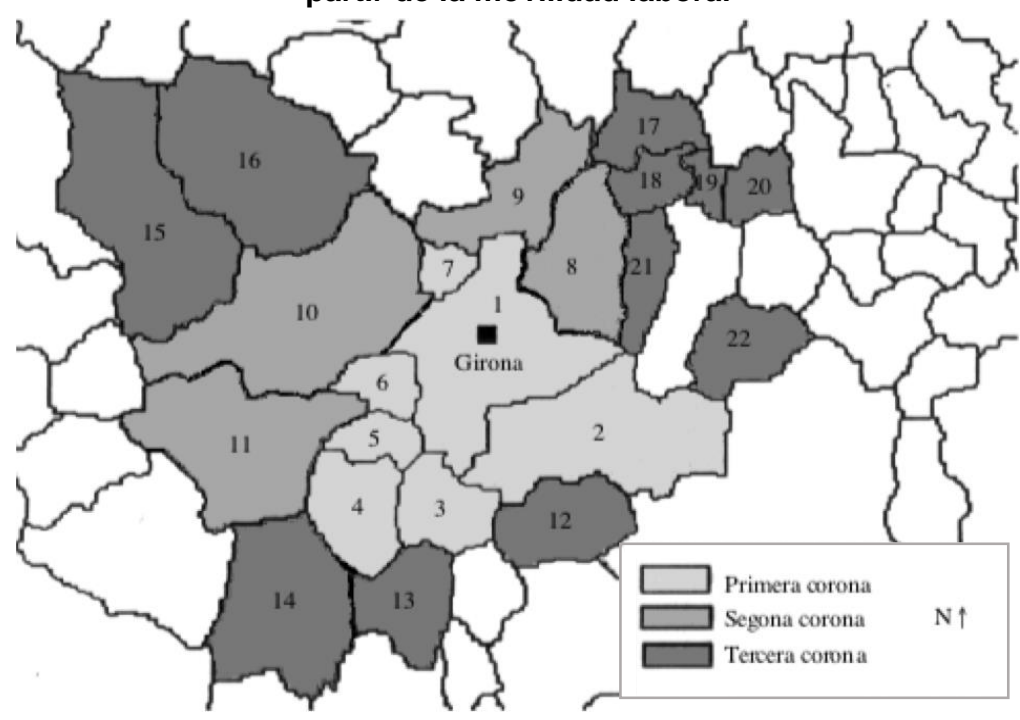

Fuente: Castañer, Vicente, Comas, Boix (1998). 
Tabla 1. Año 1986

\begin{tabular}{lll}
\hline Primera corona & Segona corona & Tercera corona \\
\hline 1. Girona & 8. Celrà & 12. Lambilles \\
2. Quart & 9. Sant Julià de Ramis & 13. Riudellots de la Selva \\
3. Fornells de la Selva & 10. Sant Gregori & 14. Vilobí d'Onyar \\
4. Aiguaviva & 11. Bescanó & 15. Sant Martí de Lémena \\
5. Vilablareix & & 16. Canet d'Adri \\
6. Salt & 17. Cervià de Ter \\
7. Sarrià de Ter & 18. Bordils \\
& 19. Sant Joan de Mollet \\
& 20. Façà \\
& & 21. Juià \\
& 22. Madremanya
\end{tabular}

Fuente: Castañer, Vicente, Comas, Boix (1998).

Figura 2. Niveles de áreas de influencia de Girona (1991).

Delimitación a partir de la movilidad laboral

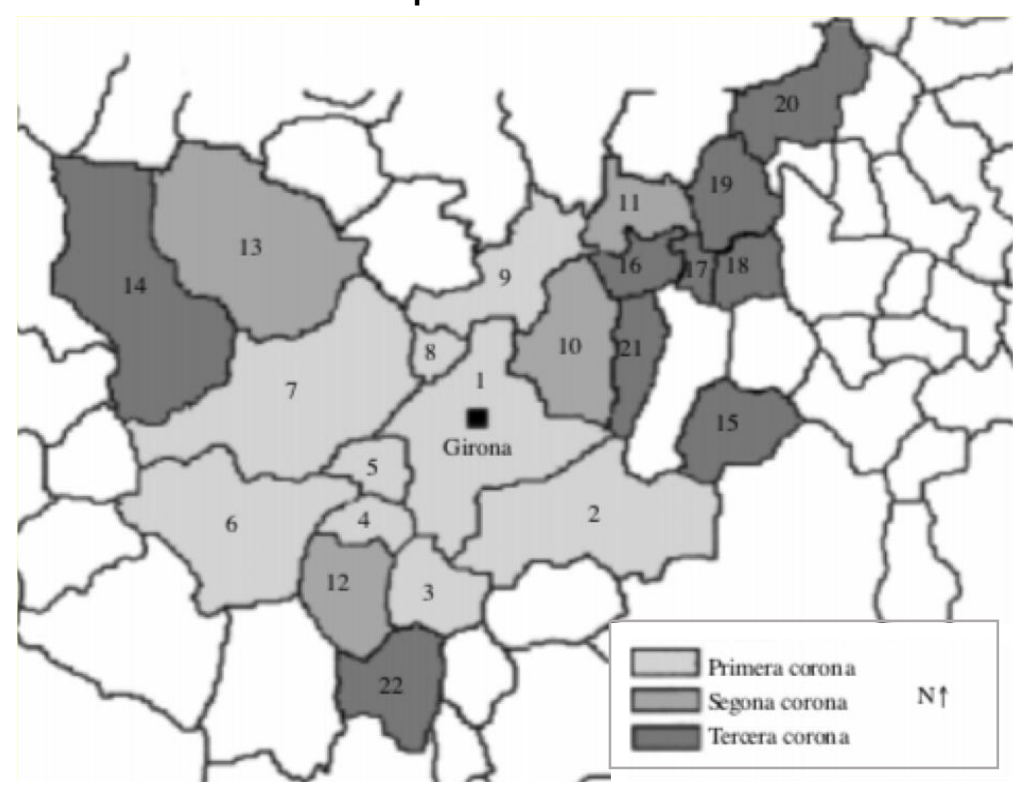

Fuente: Castañer, Vicente, Comas, Boix (1998).

Tabla 2. Año 1991

\begin{tabular}{lll}
\hline Primera corona & Segona corona & Tercera corona \\
\hline 1. Girona & 10. Celrà & 14. Sant Martí de Lémena \\
2. Quart & 11. Cervià de Ter & 15. Madremanya \\
3. Fornells de la Selva & 12. Aiguaviva & 16. Bordils \\
4. Vilablareix & 13. Canet d'Adri & 17. Sant Joan de Mollet \\
5. Salt & & 18. Flaçà \\
6. Bescanó & & 19. Sant Jordi Desvalls \\
7. Sant Gregori & 20. Vilopriu \\
8. Sarrià de Ter & 21. Juià \\
9. Sant Julià de Ramis & & 22. Riudellots de la Selva \\
\hline
\end{tabular}

Fuente: Castañer, Vicente, Comas, Boix (1998). 


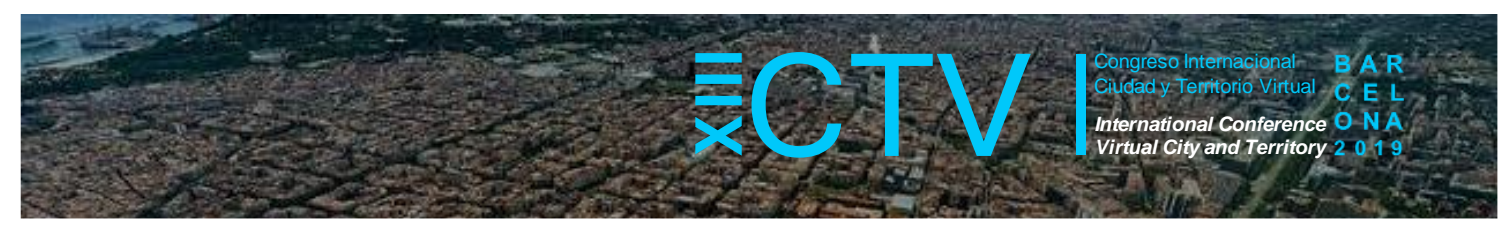

\subsubsection{Plan Director Urbanístico del Sistema Urbano de Girona}

La centralidad se trata del área que un municipio va formando según recibe flujos de movilidad laboral de otros municipios, y se establece en el momento en el que existe el primer flujo (el de número de viajes de mayor cantidad) de la Población Ocupada Residente (POR) que viene de otro municipio, con independencia de su tamaño. Es por ello por lo que, independientemente del volumen de movilidades que se den, siempre se dará algún flujo exterior. La centralidad más que un espacio homogéneo, se trata de un área que define la capacidad de atracción en su alcance máximo del municipio central, es decir, la capacidad para atraer flujos más alejados. Siguiendo estas líneas, la ciudad de Girona atrae al primer flujo de 52 municipios, obteniendo la mayor centralidad en relación con el resto de las poblaciones de las Comarcas de Girona, aunque le siguen en volumen de atracción la ciudad de Figueres y la de Olot.

En la última revisión del planeamiento urbanístico de la ciudad de Girona en el año 2000, se recopilaron diferentes propuestas sobre el alcance del área para poder hacer una propia. Así, se diferenciaron 3 niveles o coronas entorno en la ciudad de Girona: un continuo urbano con objetivo edificador y poblacional, situado desde Girona hacia los municipios de Salt, Sarrià de Ter, Fornells de la Selva, Sant Julià de Ramis y Vilablareix. Un núcleo central alrededor del continuo urbano tanto residencial como laboral, ubicado en San Gregorio, Bescanó, Aiguaviva, Riudellots de la Selva, Quart y Celrà.

Figura 3. Centralidades

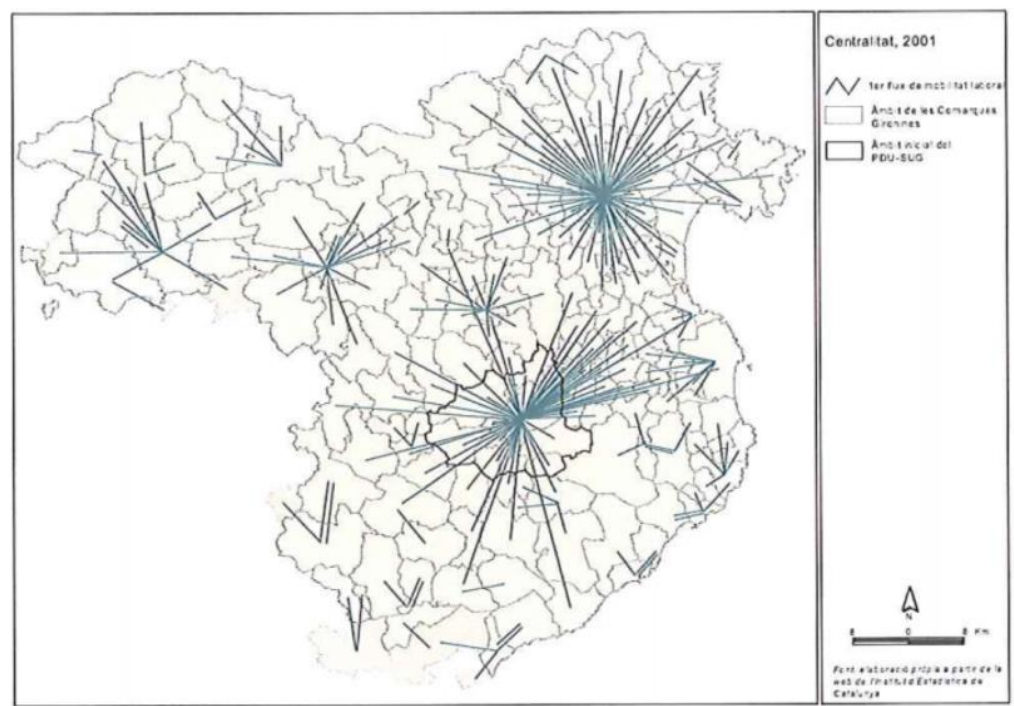

Fuente: Plan director urbanístico del sistema urbano de Girona.

\subsubsection{Continuo Urbano $>200$}

En la época del crecimiento metropolitano, los criterios que se establecieron para definir el concepto de "ciudad" fueron condicionados por el componente morfológico. Así, sobrepasados los límites de la ciudad, las aglomeraciones urbanas se definieron siguiendo unos criterios físicos. Siguiendo esto, el carácter de proximidad del crecimiento urbano se estableció como criterio para distinguir entre ciudad o entorno rural. Recogiendo por ejemplo las Naciones Unidas, las construcciones sin discontinuidad superior a 200 metros como concepto de aglomerado. Actualmente, para definir el Sistema Urbano (SU) se utiliza el método de continuo urbano utilizando como base el mapa de ocupación de suelo Corine Land Cover 2018, de las 


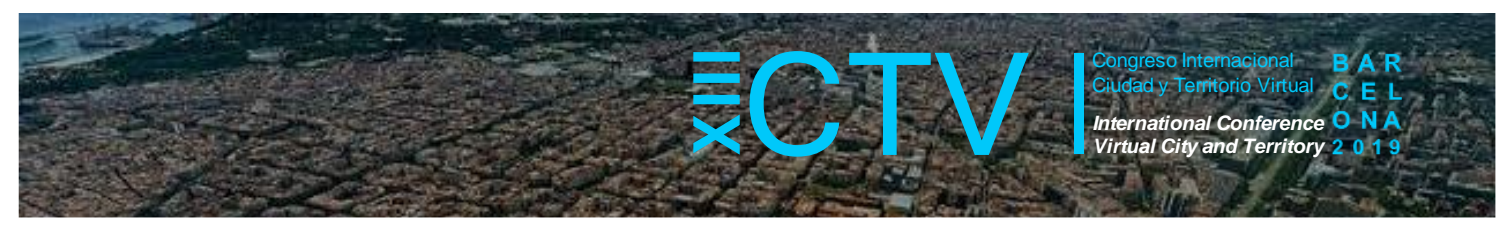

superficies artificiales (nivel 1). Habiendo obtenido el continuo urbano, se incorporan otras superficies importantes y de proximidad al continuo urbano, como por ejemplo el aeropuerto. Siendo el SU, el conjunto de todos los municipios que contienen dichas superficies. Así, en relación con el estudio del SU de Girona, queda formado por los siguientes municipios: Aiguaviva, Bescanó, Campllong, Celrá, Fornells de la Selva, Girona, Llambillas, Quart, Riudellots de la Selva, Salt, San Gregorio, San Julian de Ramis, palol de revardit, Sarriá de Ter, Vilablareix y Viloví de Oñar. Con una superficie de su continuo urbano de 49,18 km2.

Figura 4. Conjunto de municipios

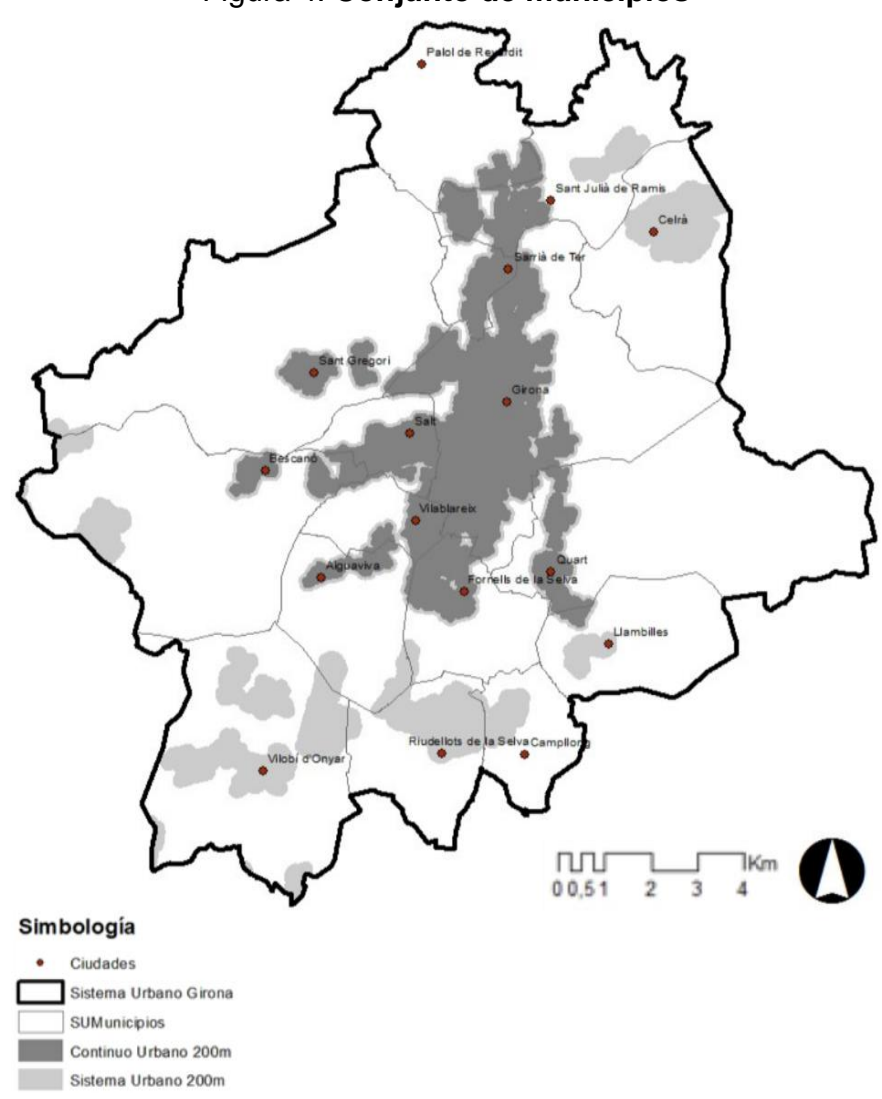

Fuente: Elaboración propia.

Tabla 3. Datos por municipios

\begin{tabular}{|l|r|r|r|}
\hline Item & Municipio & Población & Superficie \\
\hline 1 Vilobi d'Onyar & 3.196 & 32,59 & Densidad \\
\hline 2 Campllong & 535 & 8,59 & 62,28 \\
\hline 3 Riudellots de la Selva & 2.059 & 13,12 & 156,94 \\
\hline 4 Llambilles & 693 & 14,58 & 47,53 \\
\hline 5 Fornells de la Selva & 2.649 & 11,86 & 223,36 \\
\hline 6 Aiguaviva & 767 & 13,92 & 55,10 \\
\hline 7 Quart & 3.640 & 38,09 & 95,56 \\
\hline 8 Vilablareix & 2.789 & 6,17 & 452,03 \\
\hline 9 Bescanó & 4.941 & 35,92 & 137,56 \\
\hline 10 Salt & 30.622 & 6,64 & $4.611,75$ \\
\hline 11 Girona & 100.266 & 39,12 & $2.563,04$ \\
\hline 12 Sant Gregori & 3.740 & 49,17 & 76,06 \\
\hline 13 Sarrià de Ter & 5.114 & 4,16 & $1.229,33$ \\
\hline 14 Celrà & 5.390 & 19,53 & 275,99 \\
\hline 15 Sant Julià de Ramis & 3.461 & 18,8 & 184,10 \\
\hline 16 Palol de Revardit & 467 & 17,98 & 25,97 \\
\hline TOTAL & 170.329 & 330,24 & 515,77 \\
\hline
\end{tabular}

Fuente: Elaboración propia a partir de datos proporcionados por el Instituto de Estadística de Cataluña. 


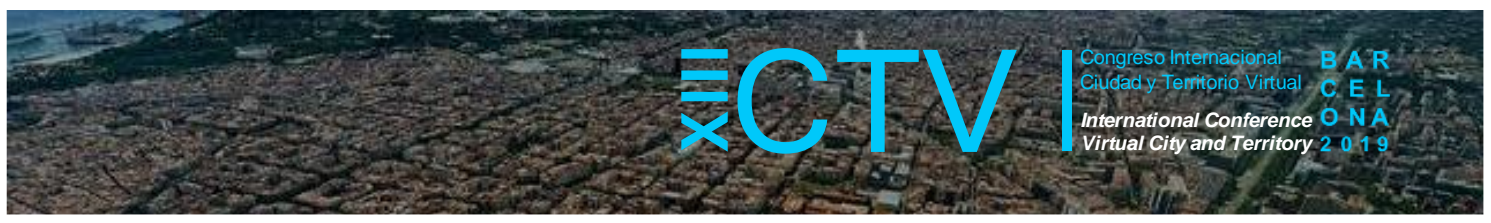

\subsection{Conociendo el territorio}

Girona (en catalán y oficialmente: Girona) es una provincia española situada al nordeste de la comunidad autónoma de Cataluña. Tiene una población de 755716 habitantes (INE 2017) y su capital es Gerona, donde vive algo más de la octava parte de la población total. Limita con las provincias de Barcelona y Lérida y con Francia, así como con el mar Mediterráneo, contando con un enclave rodeado por Francia, el municipio de Llivia.

\section{Figura 5. Imagen del continuo urbano}

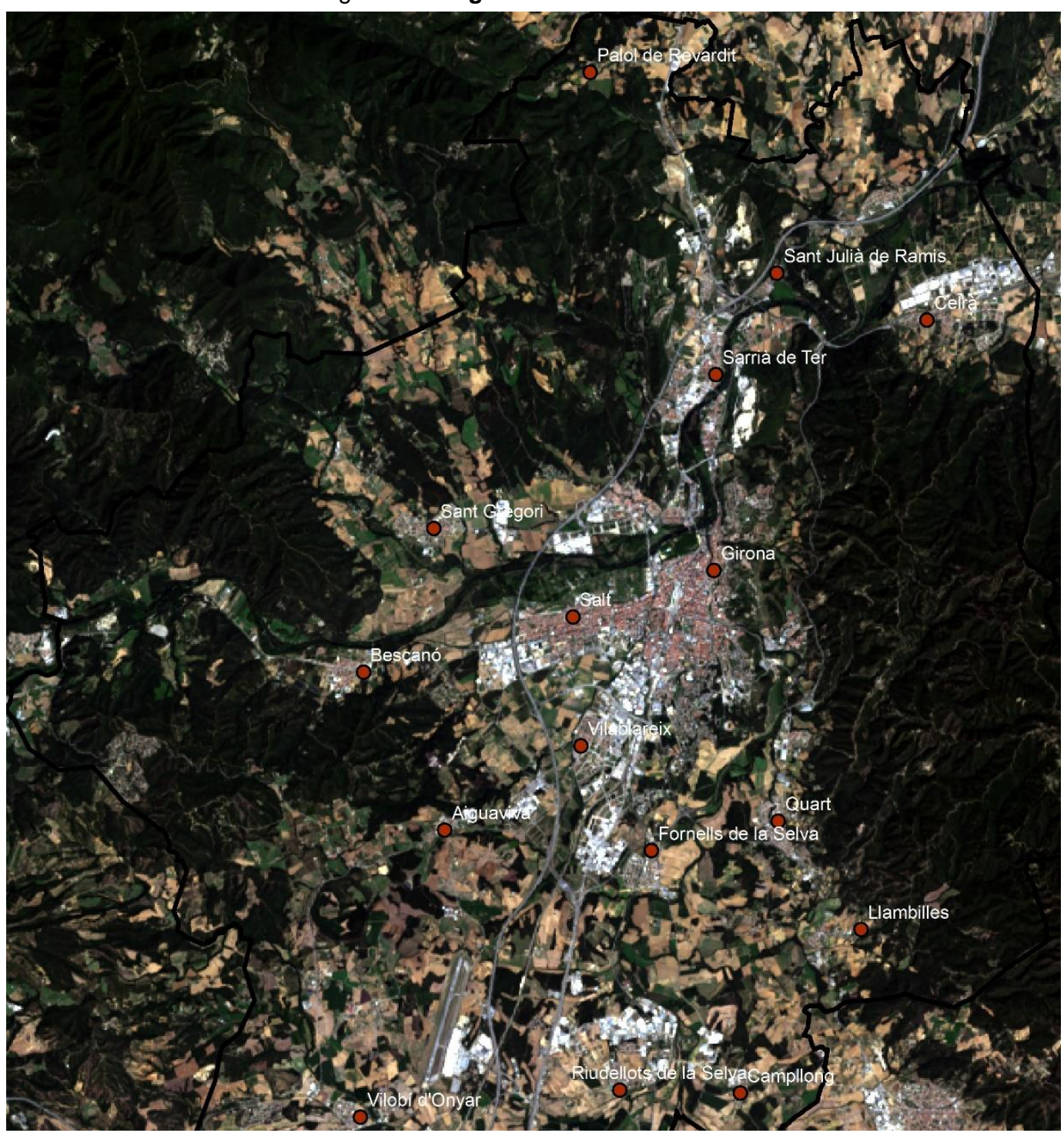

Fuente: Elaboración propia.

Para conocer el área de estudio es importante el análisis del modelo digital del terreno para conocer los distintos accidentes geográficos que puede tener el territorio y que este puede influir en el clima local. La ubicación de los distintos municipios alrededor en una meseta rodeada de montañas son uno de los principales condicionantes para la variación de su clima en las distintas épocas del año. 
Figura 6. MDT del sistema urbano de Girona

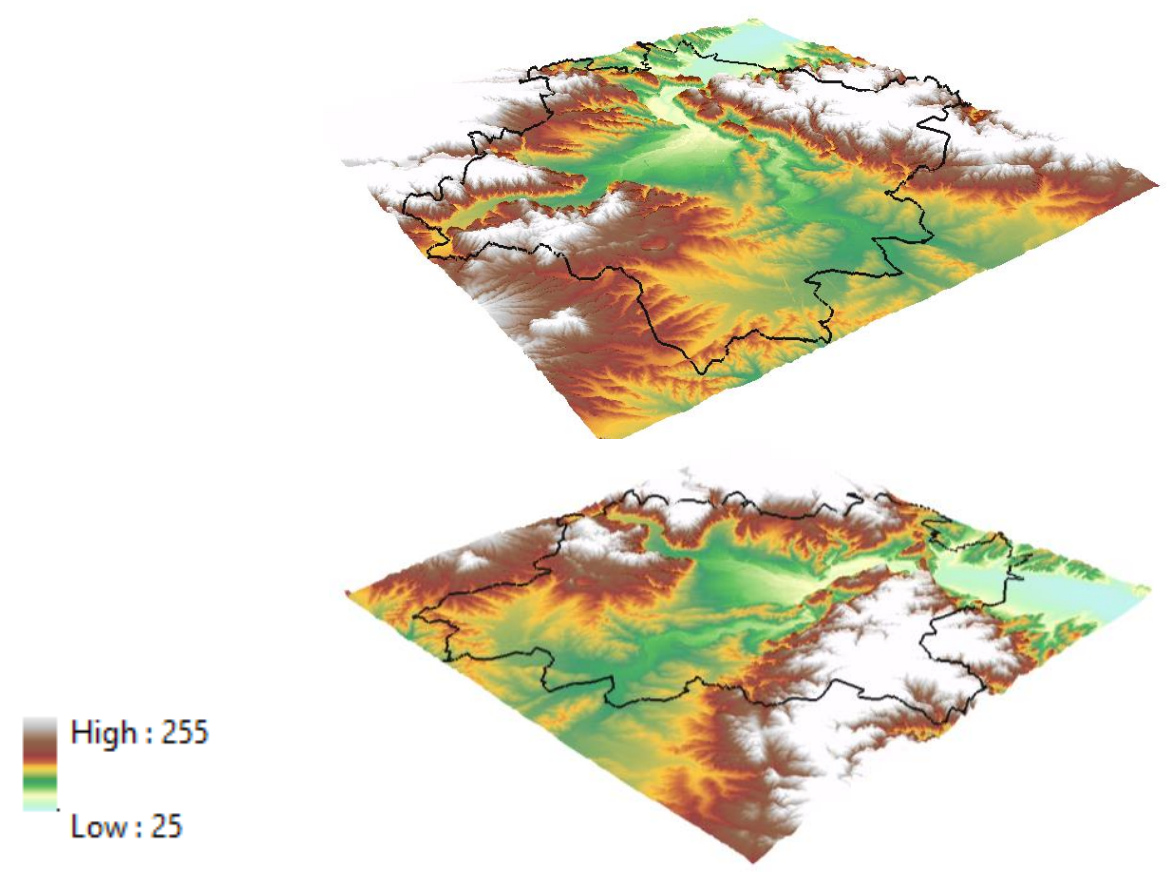

Nota: Altura en metros.

Fuente: Elaboración propia.

Figura 7. Sistema Urbano de Girona

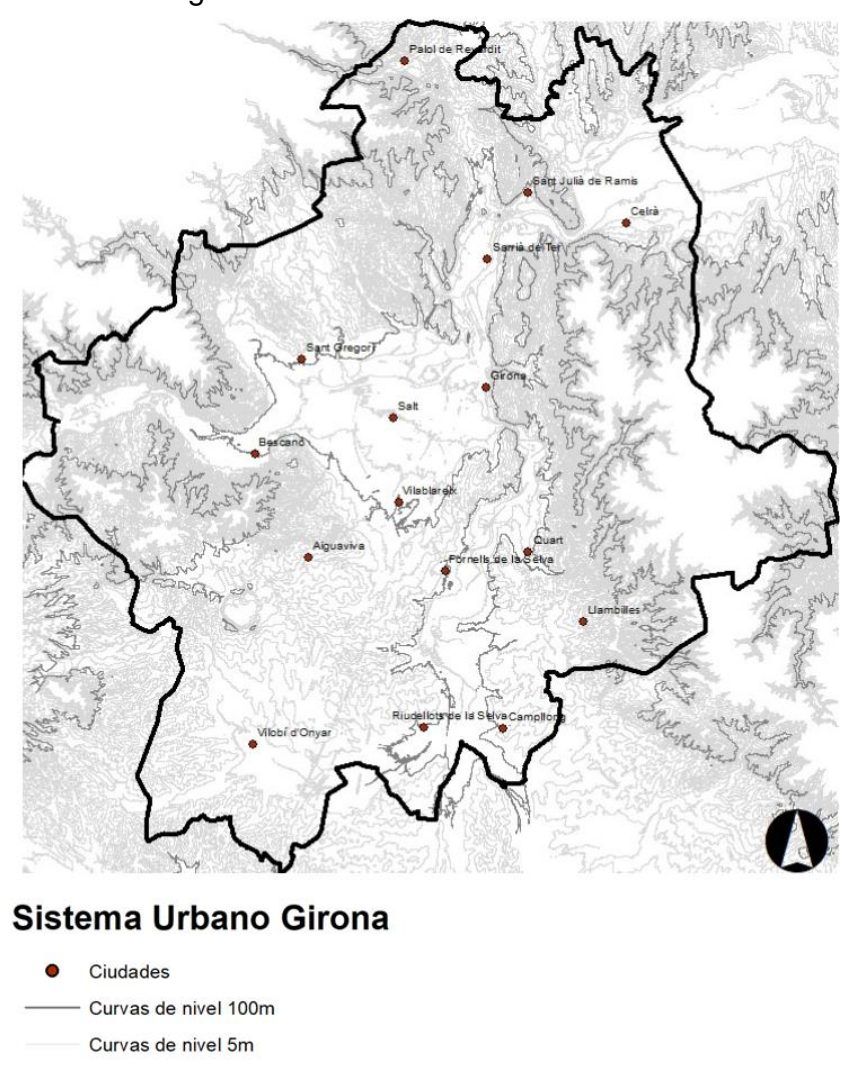

Fuente: Elaboración propia. 


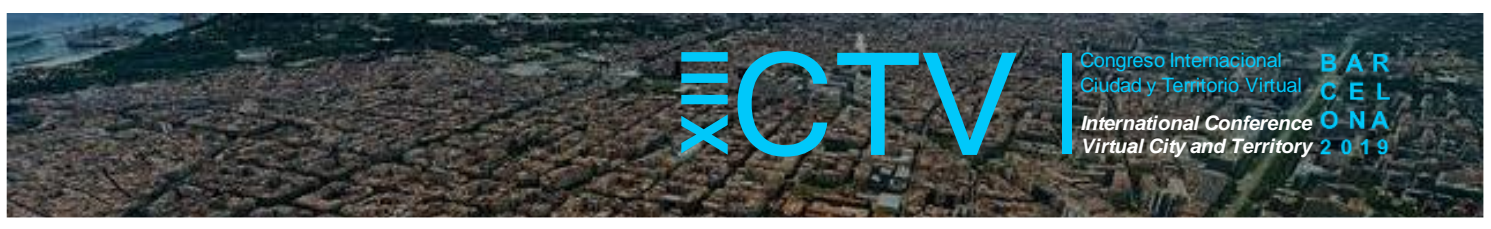

\section{2. Índices de temperatura de Suelo}

La LST (Land Surface Temperature) relaciona la radiación de la superficie y el calor entre la superficie y la atmósfera. Por tanto, uno de los principales factores que influyen en la LST es la composición de las cubiertas de suelo.

Figura 8. Diagrama de flujo para la obtención de LST

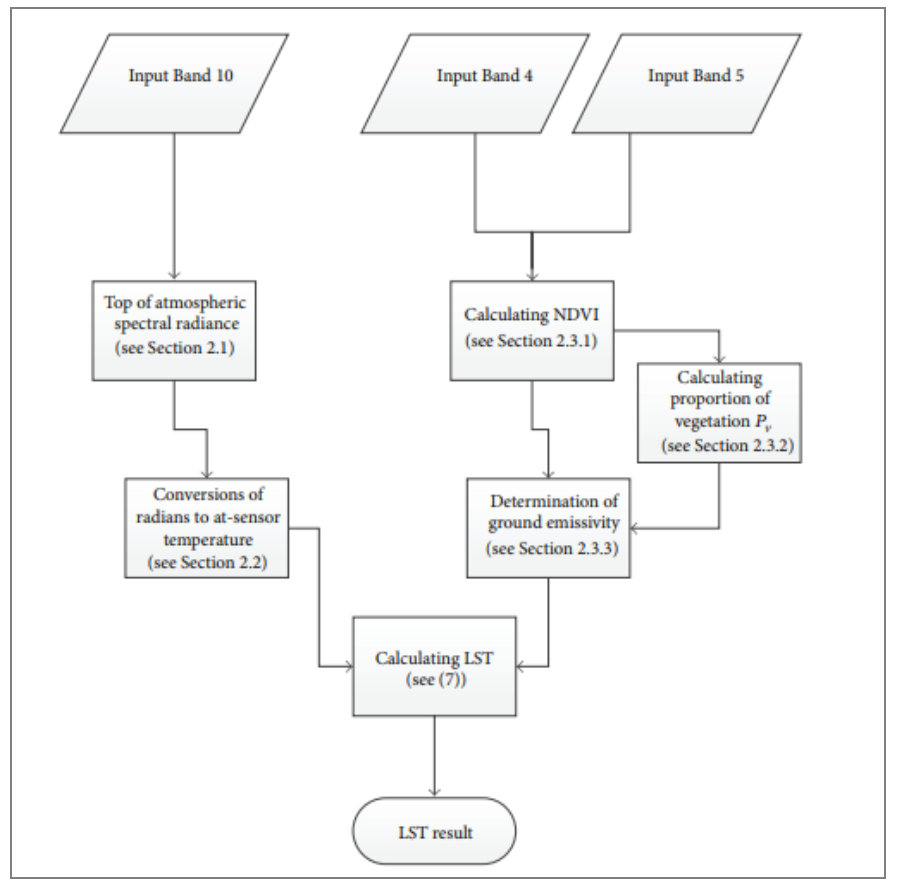

Fuente: Avdan \& Jovanovska (2016).

Para entender cómo funciona el cálculo, primero es necesario explicar de dónde viene, las ondas electromagnéticas capturan la respuesta espectral de los objetos de la superficie terrestre, (infrarrojo de onda corta (SWIR), infrarrojo de onda cercana (NIR)), materializándolas en las bandas que contienen una imagen satélite, por ejemplo; Las áreas de acumulación y el suelo desnudo reflejan más SWIR que NIR. El cuerpo de agua no se refleja en el espectro infrarrojo. En el caso de la superficie verde, la reflexión de NIR es mayor que el espectro SWIR

Figura 9. Reflectancia

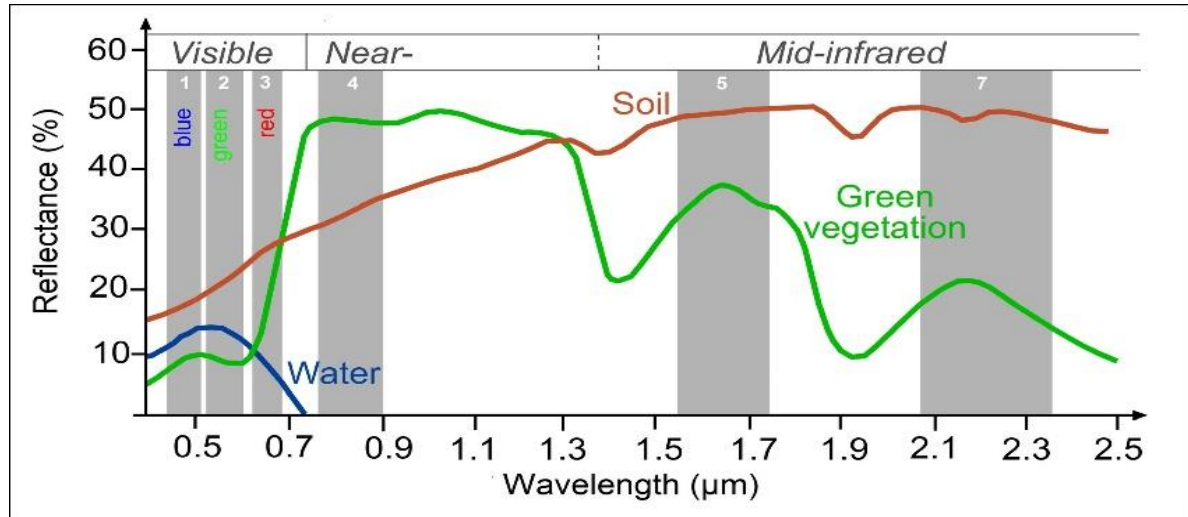

Fuente: Barsi, J., Lee K., Kvaran, G., Markham, B., and Pedelty, J. (2014). 


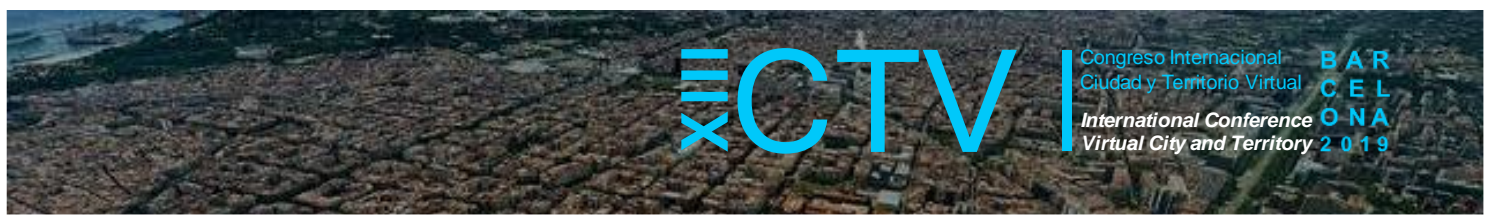

Tabla 4. Bandas de Landsat 7 y Landsat 8

\begin{tabular}{|l|ll|ll|l|}
\hline \multicolumn{2}{|l|}{ Landsat-7 ETM+ Bands $(\mu \mathrm{m})$} & \multicolumn{4}{l|}{ Landsat-8 OLI and TIRS Bands $(\mu \mathrm{m})$} \\
\hline & & & $30 \mathrm{~m}$ Coastal/Acrosol & $0.435-0.451$ & Band 1 \\
\hline Band 1 & $30 \mathrm{~m}$ Blue & $0.441-0.514$ & $30 \mathrm{~m}$ Blue & $0.452-0.512$ & Band 2 \\
\hline Band 2 & $30 \mathrm{~m}$ Green & $0.519-0.601$ & $30 \mathrm{~m}$ Green & $0.533-0.590$ & Band 3 \\
\hline Band 3 & $30 \mathrm{~m}$ Red & $0.631-0.692$ & $30 \mathrm{~m}$ Red & $0.636-0.673$ & Band 4 \\
\hline Band 4 & $30 \mathrm{~m}$ NIR & $0.772-0.898$ & $30 \mathrm{~m}$ NIR & $0.851-0.879$ & Band 5 \\
\hline Band 5 & $30 \mathrm{~m}$ SWIR-1 & $1.547-1.749$ & $30 \mathrm{~m}$ SWIR-1 & $1.566-1.651$ & Band 6 \\
\hline Band 6 & $60 \mathrm{~m}$ TIR & $10.31-12.36$ & $100 \mathrm{~m}$ TIR-I & $10.60-11.19$ & Band 10 \\
\cline { 5 - 7 } & & & $100 \mathrm{~m}$ TIR-2 & $11.50-12.51$ & Band 11 \\
\hline Band 7 & $30 \mathrm{~m}$ SWIR-2 & $2.064-2.345$ & $30 \mathrm{~m}$ SWIR-2 & $2.107-2.294$ & Band 7 \\
\hline Band 8 & $15 \mathrm{~m}$ Pan & $0.515-0.896$ & $15 \mathrm{~m}$ Pan & $0.503-0.676$ & Band 8 \\
\hline & & & $30 \mathrm{~m}$ Cirrus & $1.363-1.384$ & Band 9 \\
\hline
\end{tabular}

Fuente: Barsi, J., Lee K., Kvaran, G., Markham, B., and Pedelty, J. (2014).

EI NDVI (Normalized Difference Vegetation Index) es relevante, debido a su influencia en el balance energético de la superficie urbana. La característica principal en términos medioambientales es que la vegetación es capaz de enfriar la temperatura de la zona por lo que su cálculo nos da idea de que zona presta mejores características medioambientales. El intervalo de valores resultantes oscila entre -1 y 1

$$
N D V I=\frac{\left(\rho_{\text {NIR }}-\rho_{\text {red }}\right)}{\left(\rho_{\text {NIR }}+\rho_{\text {red }}\right)}
$$

pband: reflectancia espectral de la banda

Figura 10. Comparación de valores NDVI
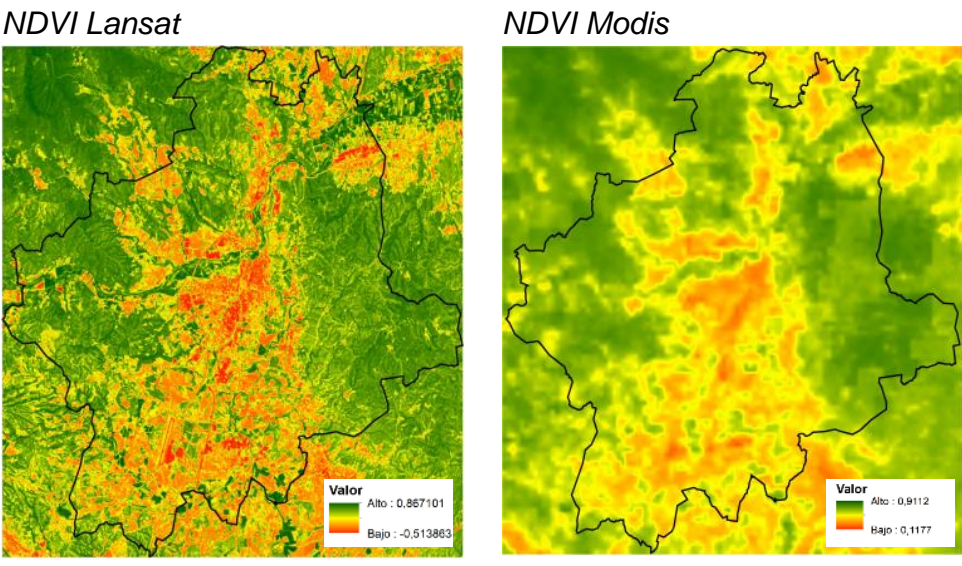

NDVI Sentinel

Fuente: Elaboración propia.

En el proceso del cálculo de la temperatura del suelo es imprescindible el cálculo del Índice de Vegetación de Diferencia Normalizada (NDVI). Este cálculo ha sido realizado usando la formula explicada anteriormente con las bandas espectrales de los satélites de Landsat, MODIS y Sentinel. Este nos demuestra la calidad del verde en nuestro sistema urbano. 


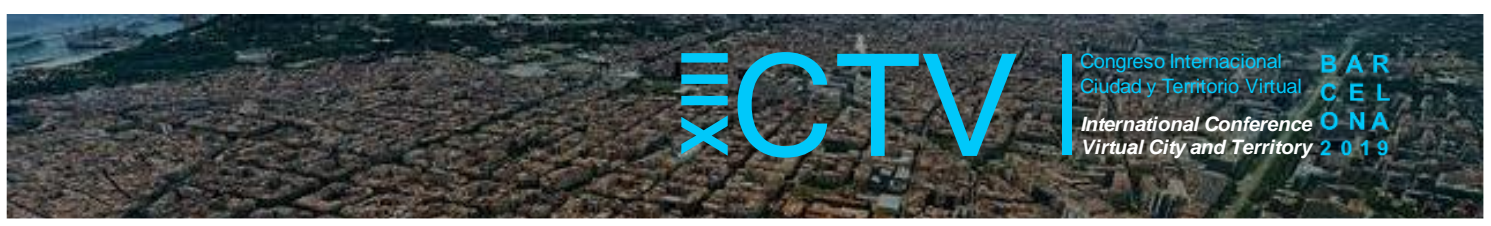

Es más visible la cantidad de verde en la imagen extraída con el satélite de LandSat con un índice inferior a 1, más precisamente 0,867 .

EI NDBI (Normalized Difference Built-up Index): Este indicador permite diferenciar zonas con superficies construidas frente a zonas naturales, por lo que es posible identificar fácilmente en el análisis zonas de vegetación, zonas desnudas y zonas edificadas pudiendo localizar los núcleos urbanos e infraestructuras como carreteras y autovías o aeropuertos. El intervalo de valores resultantes oscila entre -1 y 1

$$
N D B I=\frac{\left(\rho_{\text {SWIR } 1}-\rho_{\text {NIR }}\right)}{\left(\rho_{\text {SWIR } 1}+\rho_{\text {NIR }}\right)}
$$

pband: reflectancia espectral de la banda

FORMULA Zha, Gao, \& Ni (2003)

Figura 11. Comparación de valores NDVI
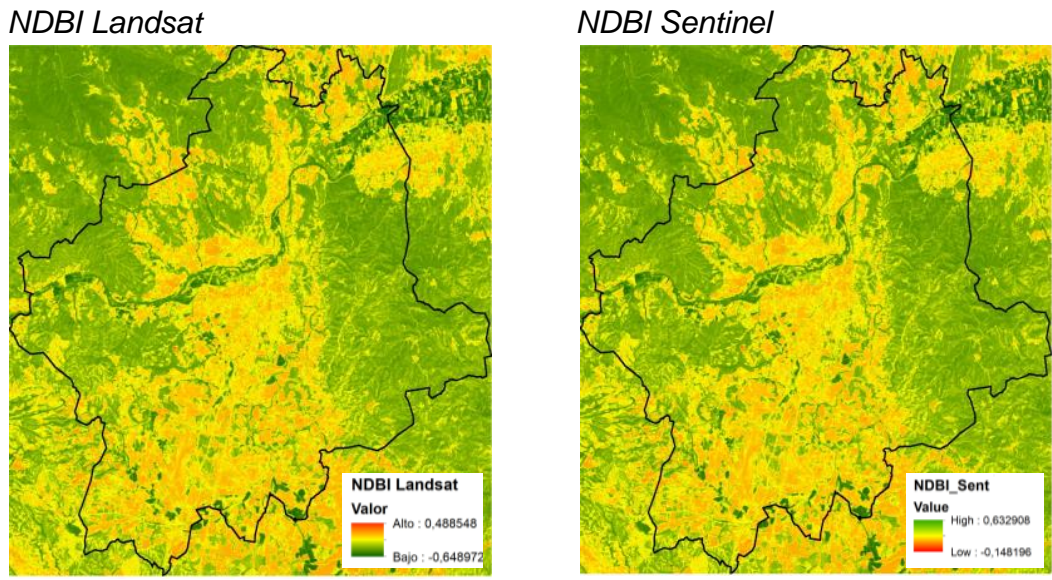

Fuente: Elaboración propia.

Una vez realizado el procesamiento de las imágenes satelitales de Landsat y Sentinel, es más evidenciable la presencia de área verde y área construida. El índice del área verde está por debajo de los -0,6 en el satélite de Landsat y por debajo de los -0,14 en el satélite de Sentinel. En este índice se puede ver más caracterizado la presencia del río y de las construcciones de baja densidad y suelos desnudos.

La fórmula de LST para Landsat es la siguiente:

$$
T_{s}=\frac{\mathrm{BT}}{\left\{1+\left[(\lambda \mathrm{BT} / \rho) \ln \varepsilon_{\lambda}\right]\right\}},
$$

donde $T s$ es el LST en grados Celsius $\left({ }^{\circ} \mathrm{C},\right)$,

BT es el sensor de radiación espectral a temperatura de brillo

$\lambda$ es la longitud de onda de la radiancia emitida (para la cual se calculará el pico y la cobertura de la longitud máxima de longitud de onda $(\lambda=10.895)$

$\varepsilon \lambda$ es la emisividad calculada por: 


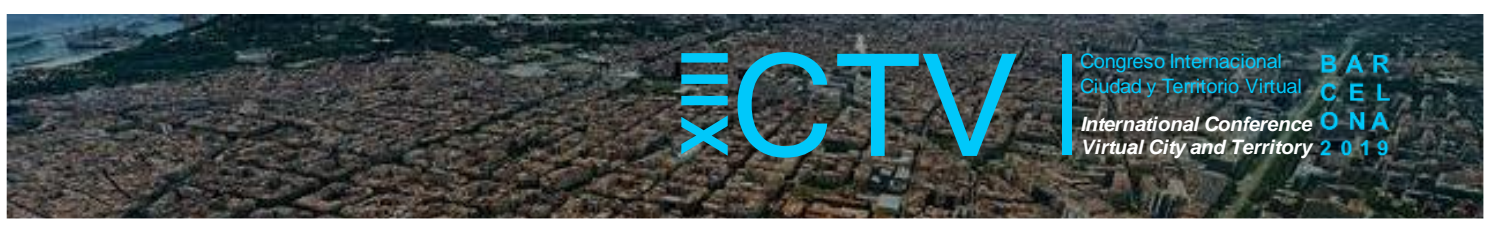

$$
\rho=\underset{\sigma}{h-}=1.438 \times 10^{-2} \mathrm{mK} \text {, }
$$

donde $\sigma$ es la constante de Boltzmann $(1.38 \times 10-23 \mathrm{~J} / \mathrm{K}), h$ es la constante de Planck $(6.626$ $\times 10-34 \mathrm{Js})$, y $c$ es la velocidad de la luz $(2.998 \times 108 \mathrm{~m} / \mathrm{s})$

\section{Figura 12. Comparación de valores LST}
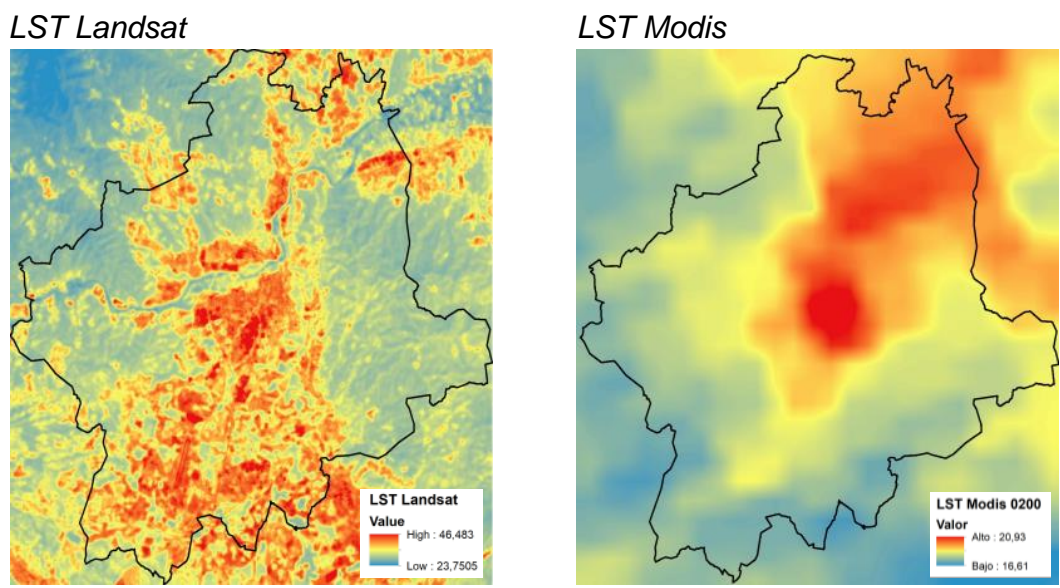

Fuente: Elaboración propia.

La imagen procesada de Landsat nos permite observar que en las áreas construidas, densas y compactas es donde se presenta las mayores temperaturas del 19 de Julio, con 46 grados Celsius. $Y$ es más evidenciable las zonas más frescas en los bosques y zonas altas del territorio.

Otra de las zonas que presentan mayor temperatura son las áreas industriales y la zona del aeropuerto que, comparando con la imagen de la noche obtenida mediante el satélite de MODIS podemos observar que estas zonas logran enfriarse en la noche con temperaturas de16-1 grados Celsius.

En MODIS ya viene procesado los valores de LST, solo es necesario multiplicar por un factor de ajuste y convertir en Celsius.

$$
\text { LST=MODIS * } 0.02-273.15
$$

Con las bandas procesadas obtenidas de MODIS en distintas horas del día es más evidente la isla de calor urbano en la zona central de nuestro sistema urbano, zona central conformada principalmente por la ciudad capital de Girona, a lo largo del día si bien estas zonas logran enfriarse hasta 15 grados Celsius, es evidente que dentro del territorio son las zonas que muy poco se enfrían.

De esta manera se comprueba por simple inspección la presencia de la isla de calor urbana, que al relacionarlo con otros datos se podrá evidenciar de mejor manera. 


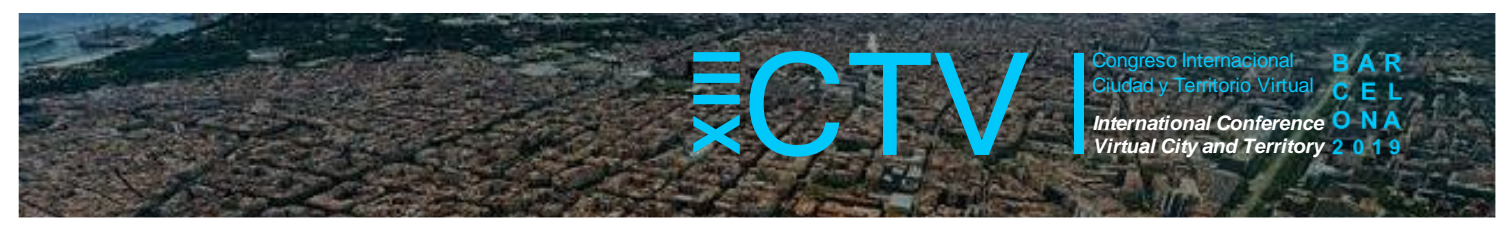

LST Modis 10:00

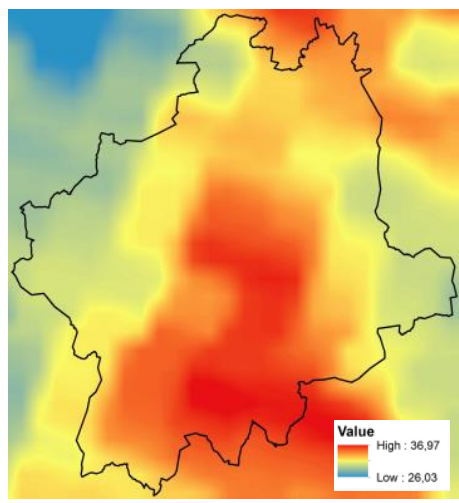

Fuente: Elaboración propia.

Figura 13. Evolución de valores LST

LST Modis 21:00

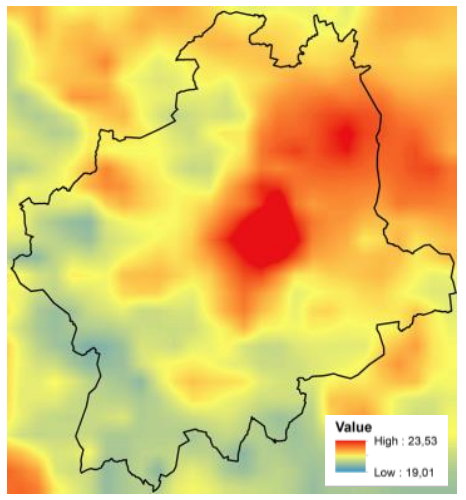

LST Modis 02:00

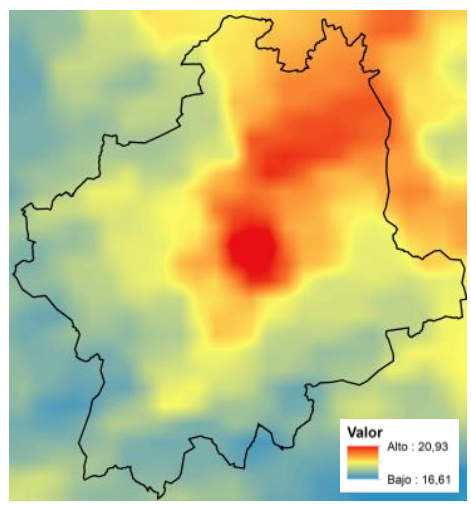

El EBBI (Enhanced Built-Up and Bareness Index): Este es el mejor índice para distinguir superficies construidas y desnudas.

$$
E B B I=\frac{\left(\rho_{\text {SWIR } 1}-\rho_{\text {NIR }}\right)}{10 \sqrt{\rho_{\text {SWIR1 } 1}+L_{\text {TIRS } 1}}}
$$

pband: reflectancia espectral de la banda

Lband: brillo espectral de la banda

FORMULA As-syakur et al. (2012)

Figura 14. Valores Ebbi

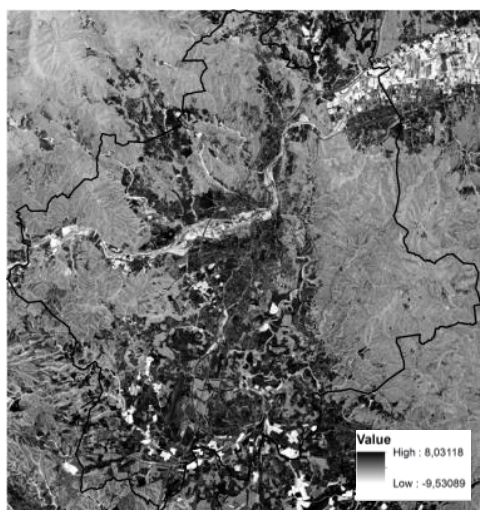

Fuente: Elaboración propia.

EI IBI (Index-based Built-up Index): El índice IBI es complejo y se deriva de los índices NDBI, Índice de vegetación ajustado al suelo (SAVI) (Huete, 1988) e índice de agua de diferencia normalizada modificada (MNDWI) (Xu, 2005; Xu, 2006). Además, el índice de vegetación de diferencia normalizada (NDVI) puede ser utilizado en el índice IBI (Rouse et al., 1973) en lugar de SAVI, cuando la cobertura de la planta supera el 30\% (Ray, 1994). El intervalo de valores resultantes oscila entre -1 y 1

$$
I B I=\frac{\left[\frac{2 \times \rho_{\text {SWIR } 1}}{\rho_{\text {SWIR1 } 1}+\rho_{\text {NIR }}}-\left(\frac{\rho_{\text {NIR }}}{\rho_{\text {NIR }}+\rho_{\text {RED }}}+\frac{\rho_{\text {GREEN }}}{\rho_{\text {GREEN }}+\rho_{\text {SWIR } 1}}\right)\right]}{\left[\frac{2 \times \rho_{\text {SWIR } 1}}{\rho_{\text {SWIR } 1}+\rho_{\text {NIR }}}+\left(\frac{\rho_{\text {NIR }}}{\rho_{\text {NIR }}+\rho_{\text {RED }}}+\frac{\rho_{\text {GREEN }}}{\rho_{\text {GREN }}+\rho_{\text {SWIR } 1}}\right)\right]}
$$

pband: reflectancia espectral de la banda 


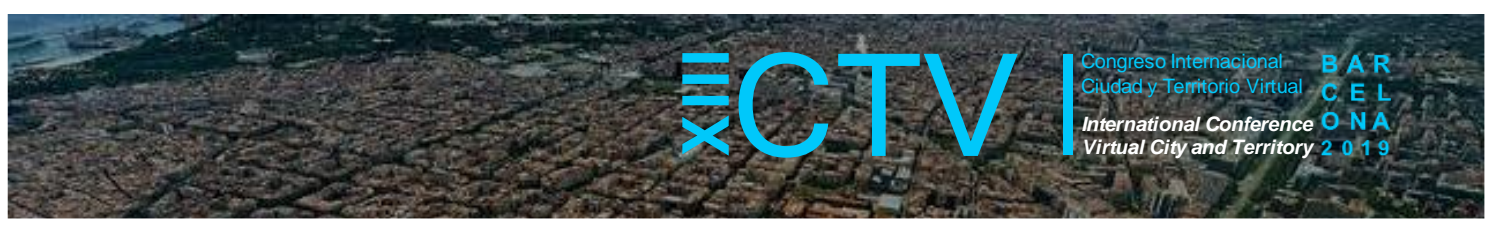

Figura 15. Valores IBBI Landsat

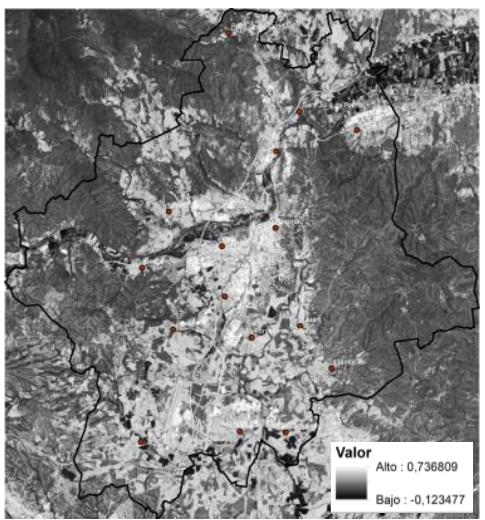

Fuente: Elaboración propia.

Con los datos obtenidos del EBBI y del $|\mathrm{B}|$ es más evidenciable la presencia de áreas construibles y la diferenciación entre áreas desnudas y áreas altamente densificadas con el índice del IBI que con el índice del EBBI.

\section{3. Índices de temperatura atmosférica}

A continuación, se muestran las estaciones meteorológicas más próximas a nuestro sistema urbano y se hace una interpolación para determinar su grado de influencia en sus proximidades y hacerse una idea la posible temperatura del aire a lo largo del territorio.

\section{Figura 16. Estaciones meteorológicas e interpolación de valores}
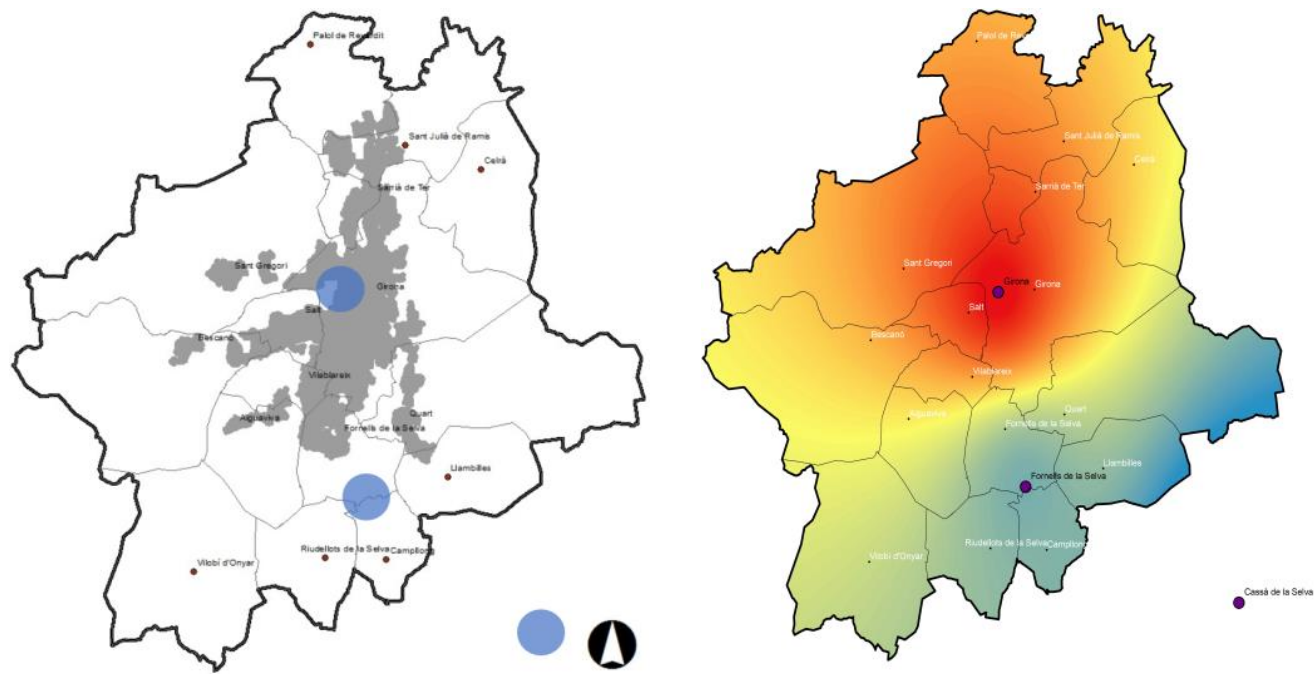

Fuente: Elaboración propia.

La estación de Cassá de Selva, ubicada apenas por fuera del sistema urbano definido previamente. Está ubicada en un sector de suelo rústico, con campo abierto y cerca de algunas áreas boscosas. Su temperatura mínima es de $15,3^{\circ} \mathrm{C}$, mientras que la máxima de $31,1^{\circ} \mathrm{C}$, con una varianza de $15,8^{\circ} \mathrm{C}$. La estación de Fornells de Selva. Dentro del sistema urbano roza el límite del continúo urbano definido previamente. Está ubicada en un sector de suelo rústico, con campo abierto y cerca de una zona urbana de tejido discontinuo. Su temperatura mínima es de $15,3^{\circ} \mathrm{C}$, mientras que la máxima de $34,3^{\circ} \mathrm{C}$, con una varianza de $19,0{ }^{\circ} \mathrm{C}$. Finalmente la 


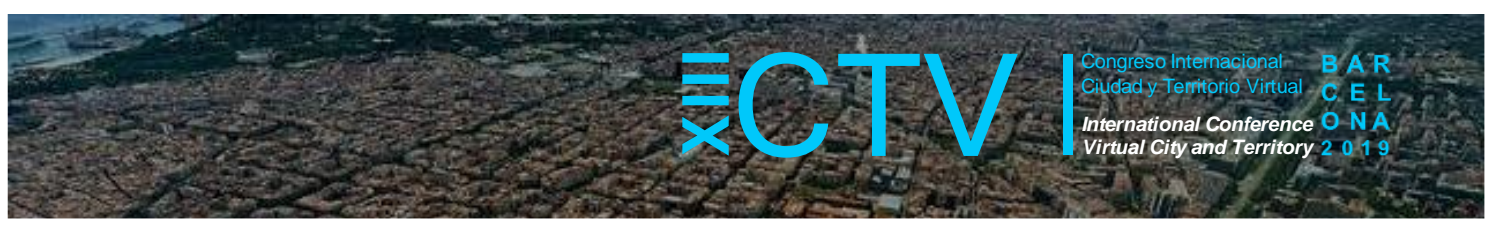

estación de Girona, cuya ubicación es la que más se asemeja a la del área de estudio de pequeña escala, la Plaza de Cataluña, que se verá más adelante. Esta estación está dentro del continuo urbano, cerca del centro, pero mantiene la característica de campo abierto, con lo cual no llega a reflejar completamente la realidad del tejido compacto. La temperatura mínima es de $16,3^{\circ} \mathrm{C}$, mientras que la máxima de $34,2^{\circ} \mathrm{C}$, con una varianza de $17,9^{\circ} \mathrm{C}$.

Comparando las tres estaciones (Tabla 5) podemos observar como a medida que se alejan del suelo urbanizado, la temperatura disminuye, calentándose menos durante el día. Mientras que la varianza es mayor en el área suburbana sugiriendo que al estar rodeada de zonas verdes se puede enfriar más durante la noche y que durante el día, al igual que la zona urbana tiende a calentarse más.

Tabla 5. Comparación de datos estaciones

\begin{tabular}{llllc}
\hline Estación & Ubicación & Mín. & Máx. & Varianza \\
Cassá & Campo & 15,3 & 31,1 & 15,8 \\
\hline Fornells & Suburbana & 15,3 & 34,3 & 19,0 \\
\hline Girona & Urbana & 16,3 & 34,2 & 17,9 \\
\hline
\end{tabular}

Fuente: Elaboración propia.

\section{Isla de Calor Urbana}

Una vez definido nuestro Sistema Urbano y realizado el análisis de la temperatura de la superficie de suelo y la temperatura atmosférica, usamos como base la calificación del suelo del mapa urbanístico de Catalunya (MUC) y la calificación del suelo del CORINE Land Cover $(\mathrm{CLC})$.

A partir de estos mapas se realizaron los ejercicios de determinar para cada calificación de suelo tanto del MUC como del CLC los diferentes indicadores estudiados como la LST de día y noche, el NDVI, el NDBI y el EBBI. Así, obtuvimos las tablas estadísticas que se exponen más adelante que nos permiten comparar el comportamiento de la temperatura según las características de la cubierta del suelo y en relación con los otros indicadores.

De la misma manera, mediante una combinación de estos dos mapas de uso de suelo y de cobertura del suelo, se obtuvo las Local Climate Zones (LCZ).

La calificación del MUC es más detallada en las zonas urbanas, definiendo densidad de los tejidos y alturas en clasificaciones como Núcleo antiguo, casas agrupadas, casas aisladas, ordenación abierta, ordenación cerrada, etc. Sin embargo, en otras clasificaciones resulta un poco ambiguo, como en el caso de los sistemas y equipamientos, ya que pueden tener una variedad de formas que corresponderían a diferentes tipos de cubiertas de suelo, como si es un espacio deportivo, un parque, una escuela o una iglesia.

De igual manera en las zonas rústicas lo que dice el plan no es necesariamente lo que se refleja en la realidad, ya que en un espacio destinado para suelo agrícola o para un futuro desarrollo urbanístico, en la actualidad puede estar ocupado por un bosque. Esto luego tendrá relevancia a la hora de determinar las Local Climate Zones. 


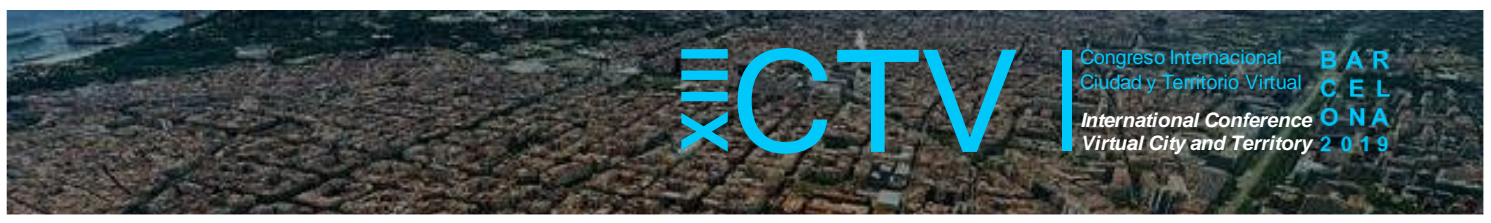

Figura 17. Usos de Suelo
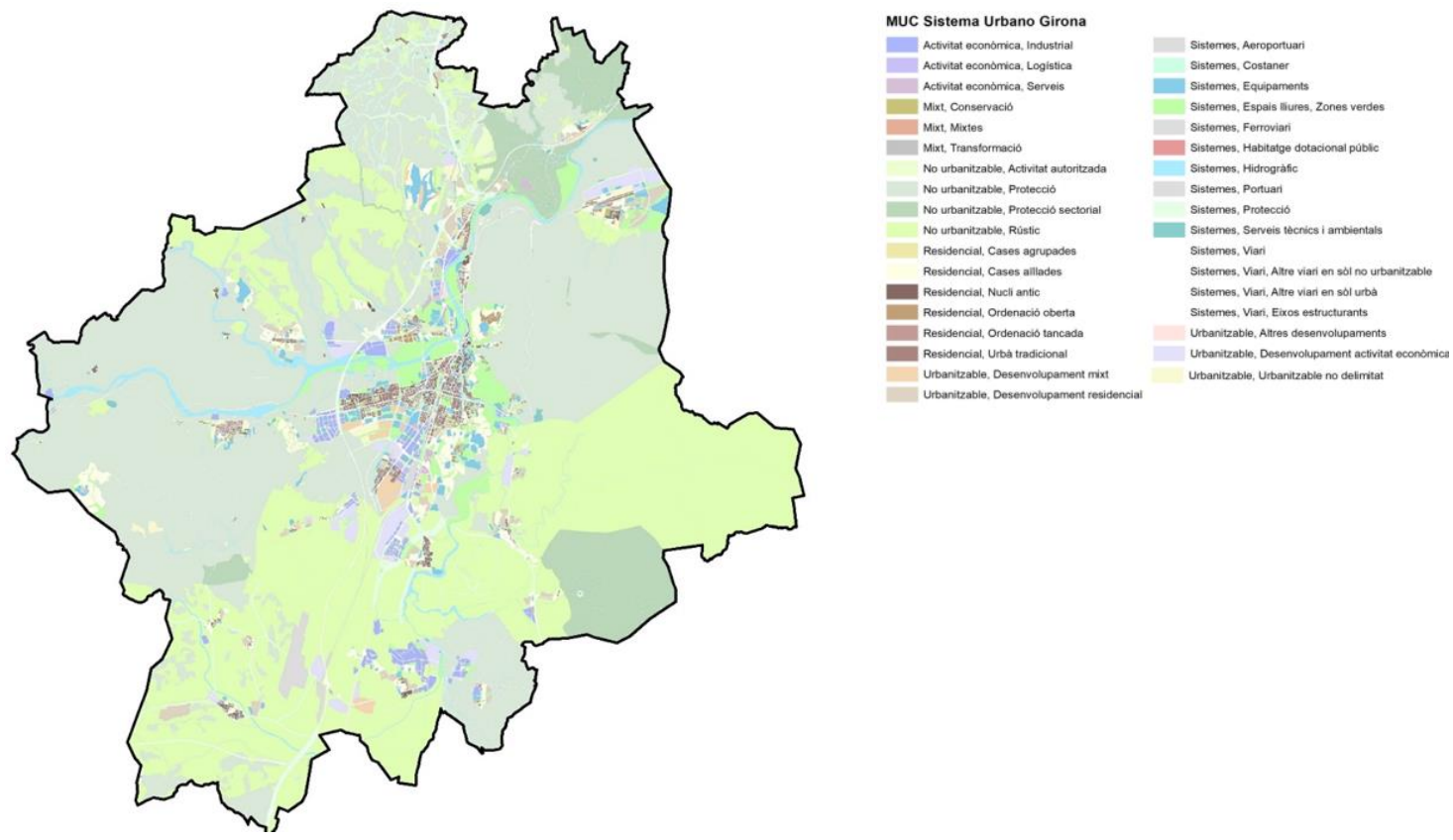

Fuente: Instituto Cartográfico de Cataluña.

En contraparte a lo que sucede con el MUC, el CLC tiene un mayor detalle en lo que se refiere a los suelos naturales o no urbanizados, con clasificaciones de matorrales, pastizales, diferentes tipos de bosques, cuerpos de agua, etc. Mientras tanto, en las zonas urbanas tiene una clasificación que distingue únicamente los tejidos continuos y discontinuos. En cuanto a lo que se refiere a redes viarias, zonas portuarias, industriales, etc., ambas clasificaciones tienen un nivel de detalle semejante.

Figura 18. Coberturas de Suelo
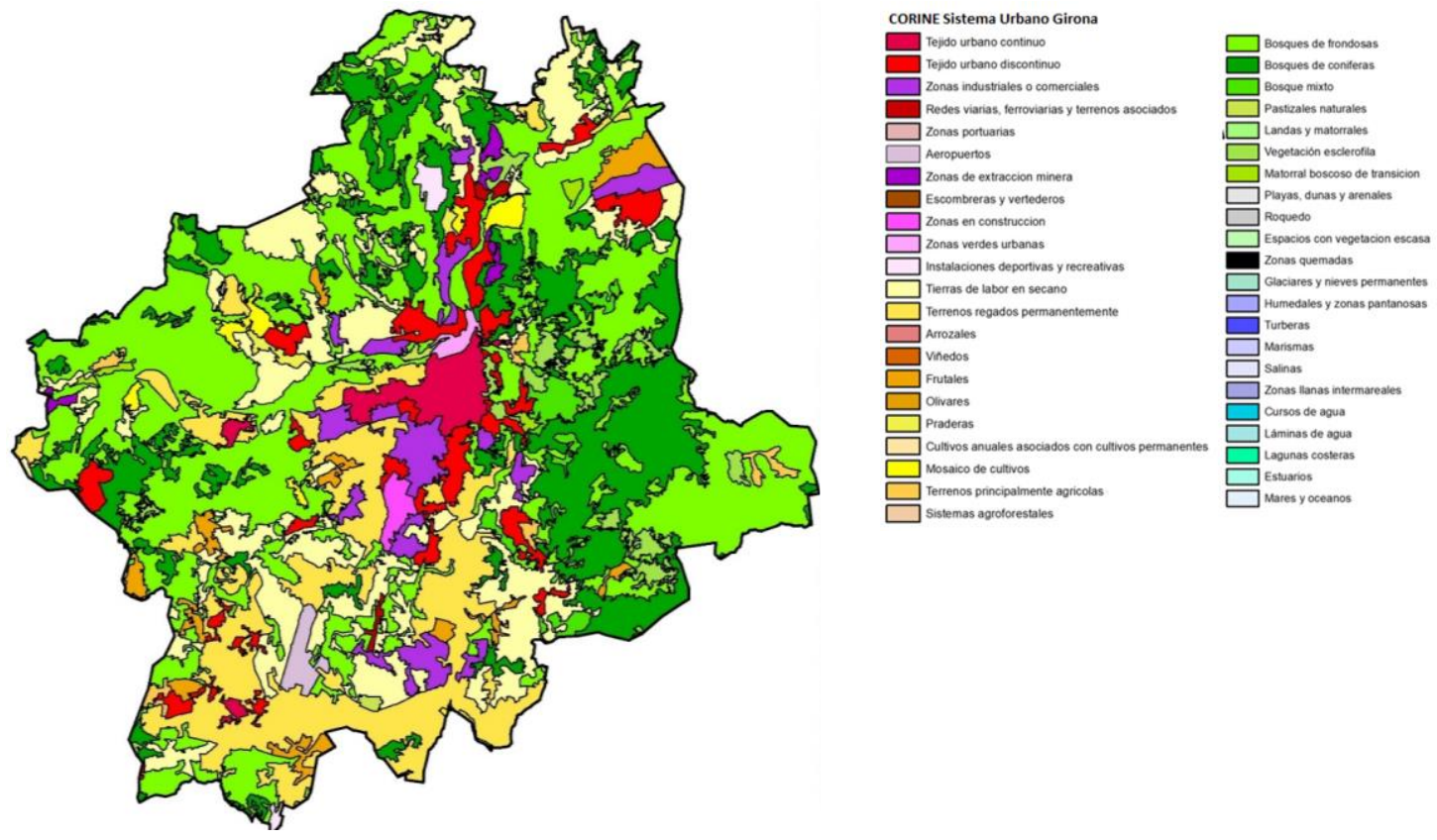

Fuente: CORINE Land Cover. 


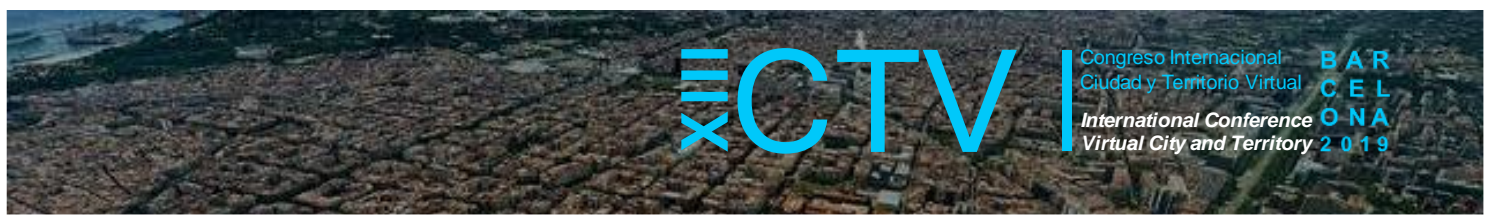

En la siguiente tabla se puede ver de manera resumida los tres grandes grupos del CLC: 1) Superficies artificiales; 2) Zonas Agrícolas; y 3) Zonas forestales con vegetación natural y espacios abiertos. Se puede ver claramente en colores rojos los indicadores que corresponden al primer grupo y como va bajando hacia los verdes y azules representativos de vegetación y bajas temperaturas respectivamente, conforme pasa a los grupos 2 y 3 . Así mismo, las superficies aumentan en ese mismo sentido, existiendo una gran cantidad de zonas forestales.

Tabla 6. Estadísticas del CORINE Land Cover

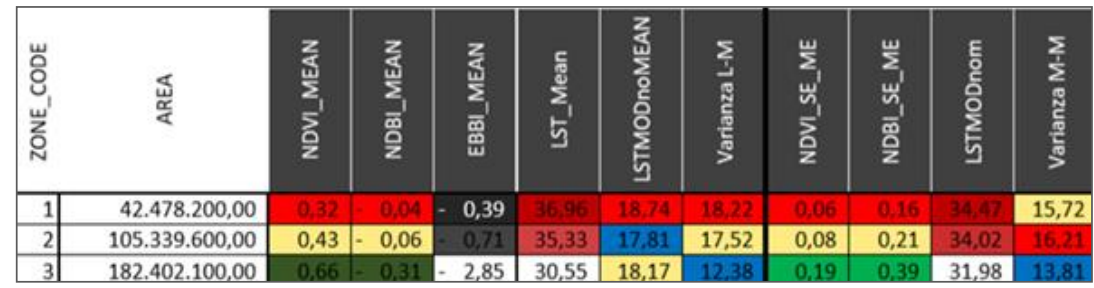

Fuente: elaboración propia en base a datos de CORINE Land cover.

En la siguiente tabla estadística se muestran las diferentes calificaciones del MUC correspondientes para cada uno de los indicadores estudiados. Han sido ordenados de acuerdo con la LST media, de mayor a menor. Se ha dividido además en dos grupos, a la izquierda los que muestran una mayor correlación entre sí y a la derecha aquellos que es menor. Se ha determinado también la varianza entre la LST de día y de noche tanto entre los satélites de Landsat y Modis, como entre Modis y Modis (en el segundo grupo).

Tabla 7. Estadísticas del MUC

\begin{tabular}{|c|c|c|c|c|c|c|c|c|c|c|c|c|}
\hline 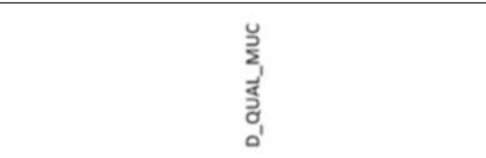 & 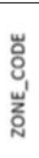 & $\underset{\frac{\alpha}{\alpha}}{\frac{\Phi}{\alpha}}$ & $\begin{array}{l}\frac{z}{5} \\
\frac{1}{2} \\
\frac{5}{z} \\
\frac{0}{z}\end{array}$ & $\begin{array}{l}\frac{z}{\alpha} \\
\frac{1}{\Sigma} \\
\frac{\bar{\alpha}}{\frac{0}{z}}\end{array}$ & $\frac{z}{\frac{z}{\Sigma}}$ & $\begin{array}{l}\frac{5}{2} \\
\frac{8}{5} \\
5\end{array}$ & 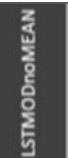 & $\begin{array}{l}\frac{\sum}{3} \\
\frac{\pi}{5} \\
\frac{5}{5} \\
\frac{5}{5}\end{array}$ & $\begin{array}{l}\frac{w}{\Sigma} \\
\frac{w}{w} \\
\frac{\sigma}{z} \\
\frac{\sigma}{z}\end{array}$ & 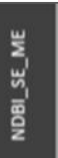 & $\begin{array}{l}\frac{0}{0} \\
\frac{0}{5} \\
\frac{5}{5}\end{array}$ & 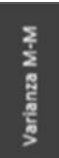 \\
\hline Activitat econvSmica, LogVzstica & 2 & 79.200 & 0,11 & 0,08 & 0,80 & 43,56 & & & 0,03 & 0,09 & 35,77 & \\
\hline Activitat econvsmica, Industrial & 1 & 3.730 .500 & 0,19 & 0,02 & 0,16 & 40,00 & 18,46 & 21,54 & 0,00 & 0,04 & & \\
\hline Sistemes, Aeroportuari & 17 & 1.223 .100 & 0,25 & 0,05 & 0,59 & 39,53 & 17,91 & 21,62 & 0,06 & 0,17 & 34,16 & 16,25 \\
\hline Residencial, Urbv' tradicional & 16 & 2.565 .900 & 0,24 & 0,05 & 0,58 & 38,31 & 19,68 & 18,63 & 0,08 & 0,21 & 32,25 & 12,57 \\
\hline Urbanitzable, Desenvolupament activitat econvsmica & 30 & 4.385 .700 & 0,27 & & 0,01 & 38,11 & 18,59 & 19,52 & 0,06 & 0,18 & 32,15 & 13,56 \\
\hline Sistemes, Habitatge dotacional pvfblic & 21 & 1.800 & 0,38 & 0,01 & 0,10 & 37,41 & & & 0,06 & 0,19 & & \\
\hline Sistemes, Viari, Altre viari en svsl urbv' & 27 & 4.118 .400 & 0,29 & 0,02 & 0,20 & 37,41 & 19,15 & 18,26 & 0,09 & 0,18 & 32,51 & 13,36 \\
\hline Residencial, Cases agrupades & 11 & 523.800 & 0,30 & & 0,03 & 37,28 & & & 0,16 & 0,35 & 31,99 & \\
\hline Residencial, Ordenaciv2 oberta & 14 & 818.100 & 0,30 & 0,01 & 0,15 & 36,99 & & & 0,16 & 0,36 & 33,02 & \\
\hline Residencial, OrdenacivZ tancada & 15 & 337.500 & 0,31 & 0,01 & 0,07 & 36,93 & & & 0,13 & 0,29 & 33,51 & \\
\hline Urbanitzable, Desenvolupament mixt & 31 & 1.622 .700 & 0,33 & $-0,02$ & 0,15 & 36,89 & 18,45 & 18,44 & 0,05 & 0,14 & & \\
\hline Residencial, Nucli antic & 13 & 837.900 & 0,32 & & 0,04 & 36,68 & 19,68 & 17,00 & 0,09 & 0,21 & 33,72 & 14,04 \\
\hline Activitat econvsmica, Serveis & 3 & 648.900 & 0,32 & 0,07 & 0,67 & 36,49 & 19,75 & 16,74 & 0,05 & 0,15 & 35,96 & 16,21 \\
\hline Sistemes, Viari, Eixos estructurants & 28 & 5.100 .300 & 0,31 & 0,06 & 0,55 & 36,37 & 18,14 & 18,23 & 0,06 & 0,14 & & \\
\hline Mixt, Transformaciv2 & 6 & 139.500 & 0,37 & 0,06 & 0,74 & 36,34 & & & 0,06 & 0,15 & & \\
\hline Urbanitzable, Desenvolupament residencial & 32 & 3.235 .500 & 0,38 & 0,04 & 0,40 & 36,34 & 17,93 & 18,41 & 0,04 & 0,11 & 35,96 & 18,03 \\
\hline Mixt, Mixtes & 5 & 31.500 & 0,38 & $-0,05$ & 0,52 & 36,32 & 19,67 & 16,65 & 0,03 & 0,09 & 35,76 & 16,09 \\
\hline Sistemes, Viari & 25 & 12.600 & 0,29 & & 0,00 & 36,31 & & & 0,07 & 0,18 & 33,04 & \\
\hline Sistemes, Serveis tv ${ }^{\circ} \mathrm{Cnics}$ i ambientals & 24 & 567.000 & 0,31 & 0,06 & 0,51 & 36,22 & 19,12 & 17,10 & 0,13 & 0,29 & 34,86 & 15,74 \\
\hline Mixt, Conservaciv2 & 4 & 146.700 & 0,39 & 0,05 & 0,57 & 36,18 & 19,95 & 16,23 & 0,06 & 0,18 & & \\
\hline Sistemes, Equipaments & 18 & 4.446 .900 & 0,37 & - 0,07 & 0,66 & 35,99 & 18,68 & 17,31 & 0,09 & 0,20 & & \\
\hline Sistemes, Ferroviari & 20 & 1.116 .000 & 0,37 & $-0,08$ & 0,76 & 35,97 & & & 0,18 & 0,41 & 34,07 & \\
\hline Residencial, Cases avøllades & 12 & 5.147 .100 & 0,41 & 0,08 & 0,80 & 35,25 & 18,18 & 17,07 & 0,12 & 0,27 & 32,42 & 14,24 \\
\hline Urbanitzable, Urbanitzable no delimitat & 33 & 941.400 & 0,46 & 0,12 & 1,30 & 34,79 & & & 0,06 & 0,15 & 33,06 & \\
\hline Sistemes, Protecciv2 & 23 & 6.344 .100 & 0,49 & 0,16 & 1,55 & 33,88 & 17,83 & 16,05 & 0,02 & 0,11 & & \\
\hline Sistemes, Espais Iliures, Zones verdes & 19 & 11.381 .400 & 0,53 & $-0,19$ & 2,01 & 33,82 & 19,23 & 14,59 & & & & \\
\hline No urbanitzable, Activitat autoritzada & 7 & 534.600 & 0,36 & $-0,08$ & 0,76 & 33,58 & 18,77 & 14,81 & 0,04 & 0,14 & 29,53 & 10,76 \\
\hline No urbanitzable, Rv Jstic & 10 & 109.575 .900 & 0,54 & $-0,18$ & 1,64 & 33,15 & 17.77 & 15,38 & 0,03 & 0,15 & 34,69 & 16,92 \\
\hline No urbanitzable, Proteccivz & 8 & 131.783 .400 & 0,60 & $-0,25$ & 2,36 & 31,60 & 18,07 & 13,53 & 0,19 & 0,48 & & \\
\hline No urbanitzable, Proteccivz sectorial & 9 & 20.640 .600 & 0,61 & 0,27 & 2,44 & 31,22 & 18,19 & 13,03 & 0,05 & 0,12 & 32,45 & 14,26 \\
\hline Sistemes, Hidrogrvtfic & 22 & 6.842 .700 & 0,66 & - 0,35 & 3,43 & 31,01 & 18,54 & 12,47 & 0,07 & 0,15 & 34,57 & 16,03 \\
\hline Urbanitzable, Altres desenvolupaments & 29 & 76.500 & 0,75 & $=0,43$ & 4,68 & 30,07 & & & 0,07 & 0,18 & 35,50 & \\
\hline Sistemes, Viari, Altre viari en sVsi no urbanitzabl & 26 & 1.260 .900 & & & 1.49 & & & & 0,10 & 0.24 & & \\
\hline
\end{tabular}

Fuente: elaboración propia en base a datos del MUC. 


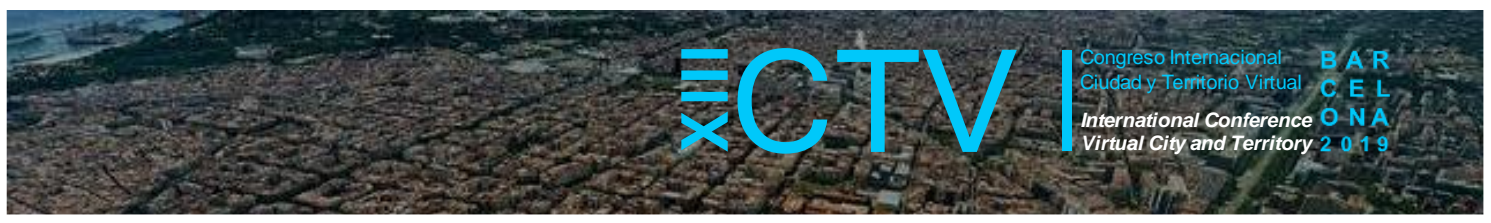

En el primer grupo, se puede ver claramente como a medida que los ámbitos son más verdes y tienen menos índice de edificación, la temperatura media disminuye y la varianza entre día y noche también, lo que muestra que a mayor verde mayor capacidad de enfriarse durante la noche tiene el suelo.

En la siguiente tabla estadística en cambio tenemos las diferentes clasificaciones del CLC correspondientes para cada uno de los indicadores estudiados. Se han ordenado y subdividido de la misma manera que se hizo en el caso del MUC. A diferencia de éste, aquí se puede apreciar que entre el primer grupo y el segundo las diferencias son menores. es decir que tenemos una correlación entre los indicadores más cercana y dentro del primer grupo el único suelo que salta a la vista como diferente es el de zonas de extracción minera, ya que a pesar de tener muy poco verde sigue siendo un espacio abierto y no construido. Es claro que en $\mathrm{NDVI}$, el NDBI, el EBBI son casi exactos y se correlacionan con la LST, demostrando lo que dice la teoría en cuanto a la temperatura que refleja cada tipo de cubierta de suelo y el impacto que éstas tienen en la capacidad de este para enfriarse en la noche, lo cual genera la isla de calor en las zonas urbanizadas.

Comparando con lo que sucede en el MUC también se hace visible que en el Corine se relacionan de mejor manera las superficies no urbanizadas, por cuanto en el MUC en muchos casos corresponden a usos variables que no representan necesariamente un único tipo de construcción u ocupación del suelo. Es por esta misma razón que para la definición de las LCZ se tomó como base el CLC y se sobrepuso a éste el MUC.

Tabla 8. Tabla estadística a partir del Corine Land Cover

\begin{tabular}{|c|c|c|c|c|c|c|c|c|c|c|c|c|}
\hline & $\begin{array}{l}\text { 山्̀ } \\
\text { 己े } \\
\text { u' } \\
\text { о }\end{array}$ & 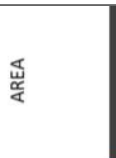 & 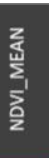 & 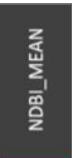 & 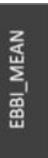 & 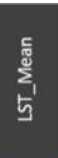 & 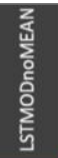 & 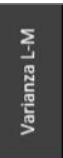 & $\begin{array}{l}\sum_{w_{1}} \\
w_{1} \\
\frac{\Sigma_{1}}{z}\end{array}$ & 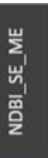 & 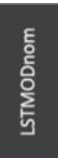 & 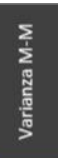 \\
\hline Aeropuertos & 124 & 1804500 & 0,27 & 0,04 & 0,42 & 39,23 & 17,81 & 21,46 & 0,04 & 0,13 & 35,58 & 17,77 \\
\hline Zonas industriales o comerciales & 121 & 12296700 & 0,27 & $-0,02$ & 0,23 & 38,43 & 18,56 & 22,01 & 0,07 & 0,18 & 34,98 & 16,42 \\
\hline Tejido urbano continuo & 111 & 6836400 & 0,27 & 0,01 & 0,10 & 37,79 & 19,90 & 22.28 & 0,04 & 0,13 & 35,41 & 15,51 \\
\hline Zonas de construcción & 133 & 1395000 & 0,33 & $-0,03$ & 0,33 & 37,01 & & & 0,06 & 0,15 & & \\
\hline Redes viarias, ferroviarias y terrenos asociados & 122 & 657000 & 0,32 & $-0,05$ & 0,50 & 36,22 & 18,48 & 20,29 & 0,04 & 0,11 & 34,41 & 15,93 \\
\hline Tejido urbano discontinuo & 112 & 15910200 & 0,37 & $-0,05$ & 0,54 & 36,20 & 18,61 & 20,99 & 0,08 & 0,18 & 33,81 & 15,20 \\
\hline Tierras de labor secano & 211 & 48411900 & 0,40 & $-0,03$ & 0,34 & 35,60 & 17,91 & 19,64 & 0,07 & 0,15 & 33,87 & 15,95 \\
\hline Terrenos regados permanentemente & 212 & 43939800 & 0,43 & - 0,08 & 0,93 & 35,48 & 17,66 & 18,76 & 0,15 & 0,34 & 34,38 & 16,72 \\
\hline Mosaico de cultivos & 242 & 3165300 & 0,43 & $-0,06$ & 0,69 & 34,54 & 18,14 & 21,18 & 0,12 & 0,25 & 31,50 & 13,36 \\
\hline Frutales & 222 & 6845400 & 0,52 & $-0,13$ & 1,52 & 33,74 & 17,96 & 18,35 & 0,07 & 0,18 & 33,34 & 15,38 \\
\hline Pastizales naturales & 321 & 517500 & 0,48 & - 0,10 & 1,21 & 33,59 & 17,53 & 14,85 & 0,08 & 0,21 & 36,27 & 18,74 \\
\hline Terrenos principalmente agrícolas,pero con importantes & 243 & 2058300 & 0,52 & $-0,12$ & 1,27 & 33,46 & 17,73 & 15,76 & 0,11 & 0,28 & 35,43 & 17,70 \\
\hline Zonas de extracción minera & 131 & 1470600 & 0,34 & $-0,08$ & 0,78 & 33,40 & 17,39 & 21,16 & 0,15 & 0,32 & 29,63 & 12,24 \\
\hline Instalaciones deportivas y recreativas & 142 & 1222200 & 0,50 & $-0,13$ & 1,37 & 33,01 & 17,67 & 17,83 & 0,09 & 0,21 & 32,85 & 15,18 \\
\hline Praderas & 231 & 918900 & 0,57 & $-0,23$ & 2,42 & 32,86 & & & 0,12 & 0,28 & & \\
\hline Matorral boscoso de transición & 324 & 1019700 & 0,60 & 0,24 & 2,80 & 31,74 & & & 0,19 & 0,40 & & \\
\hline Bosque mixto & 313 & 4557600 & 0,63 & 0,29 & 2,54 & 31,18 & 18,80 & 15,36 & 0,18 & 0,38 & 34,62 & 15,81 \\
\hline Zonas verdes urbanas & 141 & 885600 & 0,59 & 0,32 & 3,12 & 31,00 & 19,47 & 15,99 & 0,17 & 0,36 & 34,48 & 15,01 \\
\hline Vegetación esclerófila & 323 & 6965100 & 0,65 & 0,30 & 2,65 & 30,68 & 19,12 & 16,64 & 0,11 & 0,24 & 33,16 & 14,04 \\
\hline Bosques de frondosas & 311 & 115344900 & 0,67 & 0,31 & 2,99 & 30,62 & 18,08 & 16,99 & 0,19 & 0,38 & 31,71 & 13,63 \\
\hline Bosques de coniferas & 312 & 53997300 & 0,64 & 0,31 & 2,64 & 30,27 & 18,24 & 16,27 & 0,14 & 0,34 & 32,25 & 14,01 \\
\hline
\end{tabular}

Fuente: Elaboración propia en base a datos de CORINE Land cover.

\section{Local Climate Zones}

En la tabla del MUC es interesante la diferencia en las varianzas, que llega a ser de $9,07^{\circ} \mathrm{C}$. Las más altas en la parte alta, la actividad industrial y aeropuerto, mientras que abajo los sistemas hidrográficos y los no urbanizables de protección. 


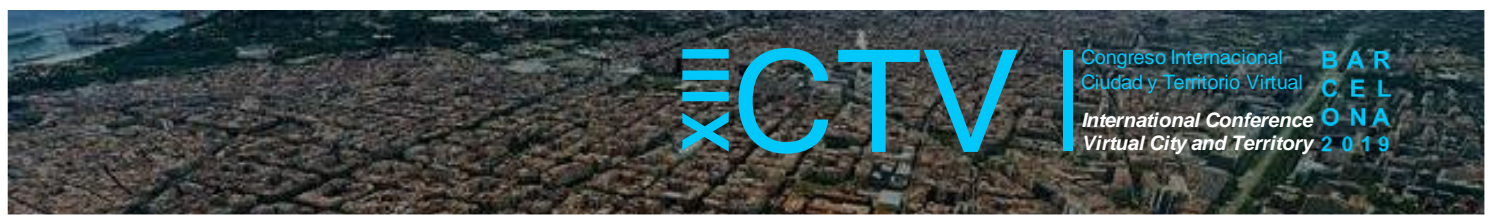

Tabla 9. Estadísticas comparadas del MUC

\begin{tabular}{|c|c|c|c|c|c|}
\hline $\begin{array}{l}\text { U } \\
\sum_{1} \\
\frac{1}{\delta} \\
\sigma_{0} \\
0^{\prime}\end{array}$ & 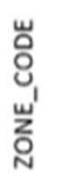 & 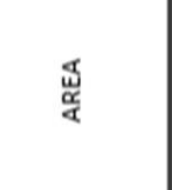 & $\begin{array}{l}\frac{z}{\alpha} \\
\frac{1}{2} \\
\frac{5}{5} \\
\frac{\partial}{z}\end{array}$ & 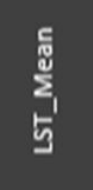 & 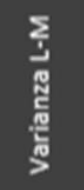 \\
\hline Activitat econvsmica, LogV₹stica & 2 & 79.200 & 0,11 & 43,56 & \\
\hline Activitat econvsmica, Industrial & 1 & 3.730 .500 & 0,19 & 40,00 & 21,54 \\
\hline Sistemes, Aeroportuari & 17 & 1.223 .100 & 0,25 & 39,53 & 21,62 \\
\hline Residencial, Urbv + tradicional & 16 & 2.565 .900 & 0,24 & 38,31 & 18,63 \\
\hline Urbanitzable, Desenvolupament activitat econvsmica & 30 & 4.385 .700 & 0,27 & 38,11 & 19,52 \\
\hline Sistemes, Habitatge dotacional pvjblic & 21 & 1.800 & 0,38 & 37,41 & \\
\hline Sistemes, Viari, Altre viari en svsl urbv ${ }^{\dagger}$ & 27 & 4.118 .400 & 0,29 & 37,41 & 18,26 \\
\hline Residencial, Cases agrupades & 11 & 523.800 & 0,30 & 37,28 & \\
\hline Residencial, Ordenaciv $\geq$ oberta & 14 & 818.100 & 0,30 & 36,99 & \\
\hline Residencial, Ordenaciv $\geq$ tancada & 15 & 337.500 & 0,31 & 36,93 & \\
\hline Urbanitzable, Desenvolupament mixt & 31 & 1.622 .700 & 0,33 & 36,89 & 18,44 \\
\hline Residencial, Nucli antic & 13 & 837.900 & 0,32 & 36,68 & 17,00 \\
\hline Activitat econvsmica, Serveis & 3 & 648.900 & 0,32 & 36,49 & 16,74 \\
\hline Sistemes, Viari, Eixos estructurants & 28 & 5.100 .300 & 0,31 & 36,37 & 18,23 \\
\hline Mixt, Transformaciv $\geq$ & 6 & 139.500 & 0,37 & 36,34 & \\
\hline Urbanitzable, Desenvolupament residencial & 32 & 3.235 .500 & 0,38 & 36,34 & 18,41 \\
\hline Mixt, Mixtes & 5 & 31.500 & 0,38 & 36,32 & 16,65 \\
\hline Sistemes, Viari & 25 & 12.600 & 0,29 & 36,31 & \\
\hline Sistemes, Serveis $\mathrm{tv}^{\circ} \mathrm{cnics} i$ ambientals & 24 & 567.000 & 0,31 & 36,22 & 17,10 \\
\hline Mixt, Conservaciv 2 & 4 & 146.700 & 0,39 & 36,18 & 16,23 \\
\hline Sistemes, Equipaments & 18 & 4.446 .900 & 0,37 & 35,99 & 17,31 \\
\hline Sistemes, Ferroviari & 20 & 1.116 .000 & 0,37 & 35,97 & \\
\hline Residencial, Cases avøllades & 12 & 5.147 .100 & 0,41 & 35,25 & 17,07 \\
\hline Urbanitzable, Urbanitzable no delimitat & 33 & 941.400 & 0,46 & 34,79 & \\
\hline Sistemes, Protecciv $\geq$ & 23 & 6.344 .100 & 0,49 & 33,88 & 16,05 \\
\hline Sistemes, Espais lliures, Zones verdes & 19 & 11.381 .400 & 0,53 & 33,82 & 14,59 \\
\hline No urbanitzable, Activitat autoritzada & 7 & 534.600 & 0,36 & 33,58 & 14,81 \\
\hline No urbanitzable, Rvsstic & 10 & 109.575 .900 & 0,54 & 33,15 & 15,38 \\
\hline No urbanitzable, Protecciv $\geq$ & 8 & 131.783 .400 & 0,60 & 31,60 & 13,53 \\
\hline No urbanitzable, Proteccivz sectorial & 9 & 20.640 .600 & 0,61 & 31,22 & 13,03 \\
\hline Sistemes, Hidrogrvtfic & 22 & 6.842 .700 & 0,66 & 31,01 & 12,47 \\
\hline Urbanitzable, Altres desenvolupaments & 29 & 76.500 & 0,75 & 30,07 & \\
\hline Sistemes, Viari, Altre viari en sVsı no urbanitzabl & 26 & 1.260 .900 & & & \\
\hline
\end{tabular}

Fuente: elaboración propia en base a datos del MUC.

A continuación, se presentan las tablas resumidas del MUC y del CLC, de donde se extrajo los indicadores más representativos: el NDVI, la LST y la varianza de LST de día (Landsat) y noche (Modis). Se puede ver como en la parte superior en rojo con LSTs que superan los $40^{\circ} \mathrm{C}$ coinciden en ambas tablas con mayores temperaturas, mayores varianzas y menor cantidad de verde calificaciones como actividades industriales y logísticas, sistemas aeroportuarios, residencial tradicional y coberturas de suelo como aeropuertos, zonas industriales 0 comerciales y tejido continuo. otras clasificaciones urbanas como casas agrupadas, ordenación abierta y cerrada y el núcleo antiguo aparecen más abajo en la tabla con NDVI cercano al 0,30 y temperaturas de $37^{\circ} \mathrm{C}$ aprox., mientras en las coberturas el Tejido urbano discontinuo a pesar de verse más rojo tiene un NDVI mayor (de 0,37 ) y una LST menor, de $36,20^{\circ} \mathrm{C}$. Aparecen con mayores varianzas las zonas mineras y el mosaico de cultivos y con las menores los pastizales naturales, zonas verdes urbanas y bosques. 
Tabla 10. Estadísticos

\begin{tabular}{|c|c|c|c|c|c|}
\hline & 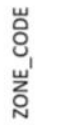 & 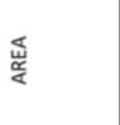 & $\begin{array}{l}\frac{z}{\alpha} \\
\frac{1}{\Sigma_{1}} \\
\frac{z_{1}}{z}\end{array}$ & $\begin{array}{l}\text { 대 } \\
\sum_{1}^{\infty} \\
5\end{array}$ & $\begin{array}{l}\sum_{j} \\
\stackrel{\mathbb{Z}}{ } \\
\frac{\Phi}{5} \\
>\end{array}$ \\
\hline Aeropuertos & 124 & 1804500 & 0,27 & 39,23 & 21,46 \\
\hline Zonas industriales o comerciales & 121 & 12296700 & 0,27 & 38,43 & 22,01 \\
\hline Tejido urbano continuo & 111 & 6836400 & 0,27 & 37,79 & 22.28 \\
\hline Zonas de construcción & 133 & 1395000 & 0,33 & 37,01 & \\
\hline Redes viarias, ferroviarias y terrenos asociados & 122 & 657000 & 0,32 & 36,22 & 20,29 \\
\hline Tejido urbano discontinuo & 112 & 15910200 & 0,37 & 36,20 & 20,99 \\
\hline Tierras de labor secano & 211 & 48411900 & 0,40 & 35,60 & 19,64 \\
\hline Terrenos regados permanentemente & 212 & 43939800 & 0,43 & 35,48 & 18,76 \\
\hline Mosaico de cultivos & 242 & 3165300 & 0,43 & 34,54 & 21,18 \\
\hline Frutales & 222 & 6845400 & 0,52 & 33,74 & 18,35 \\
\hline Pastizales naturales & 321 & 517500 & 0,48 & 33,59 & 14,85 \\
\hline Terrenos principalmente agrícolas,pero con import & 243 & 2058300 & 0,52 & 33,46 & 15,76 \\
\hline Zonas de extracción minera & 131 & 1470600 & 0,34 & 33,40 & 21,16 \\
\hline Instalaciones deportivas y recreativas & 142 & 1222200 & 0,50 & 33,01 & 17,83 \\
\hline Praderas & 231 & 918900 & 0,57 & 32,86 & \\
\hline Matorral boscoso de transición & 324 & 1019700 & 0,60 & 31,74 & \\
\hline Bosque mixto & 313 & 4557600 & 0,63 & 31,18 & 15,36 \\
\hline Zonas verdes urbanas & 141 & 885600 & 0,59 & 31,00 & 15,99 \\
\hline Vegetación esclerófila & 323 & 6965100 & 0,65 & 30,68 & 16,64 \\
\hline Bosques de frondosas & 311 & 115344900 & 0,67 & 30,62 & 16,99 \\
\hline Bosques de coniferas & 312 & 53997300 & 0,64 & 30,27 & 16,27 \\
\hline
\end{tabular}

Fuente: Elaboración propia

Según Arrizabalaga et al., la determinación de los climatopes se realizará en base a los índices obtenidos anteriormente, priorizándolos datos de áreas urbanas verdes, la información respecto a la construcción existente, la topografía del territorio obtenida mediante el MDT, yla información obtenida de las distintas estaciones meteorológicas que existen en el lugar. Proceso que ha sido llevado a cabo para la categorización de los Local Climate Zones.

Figura 19. Proceso de determinación de los climatopes

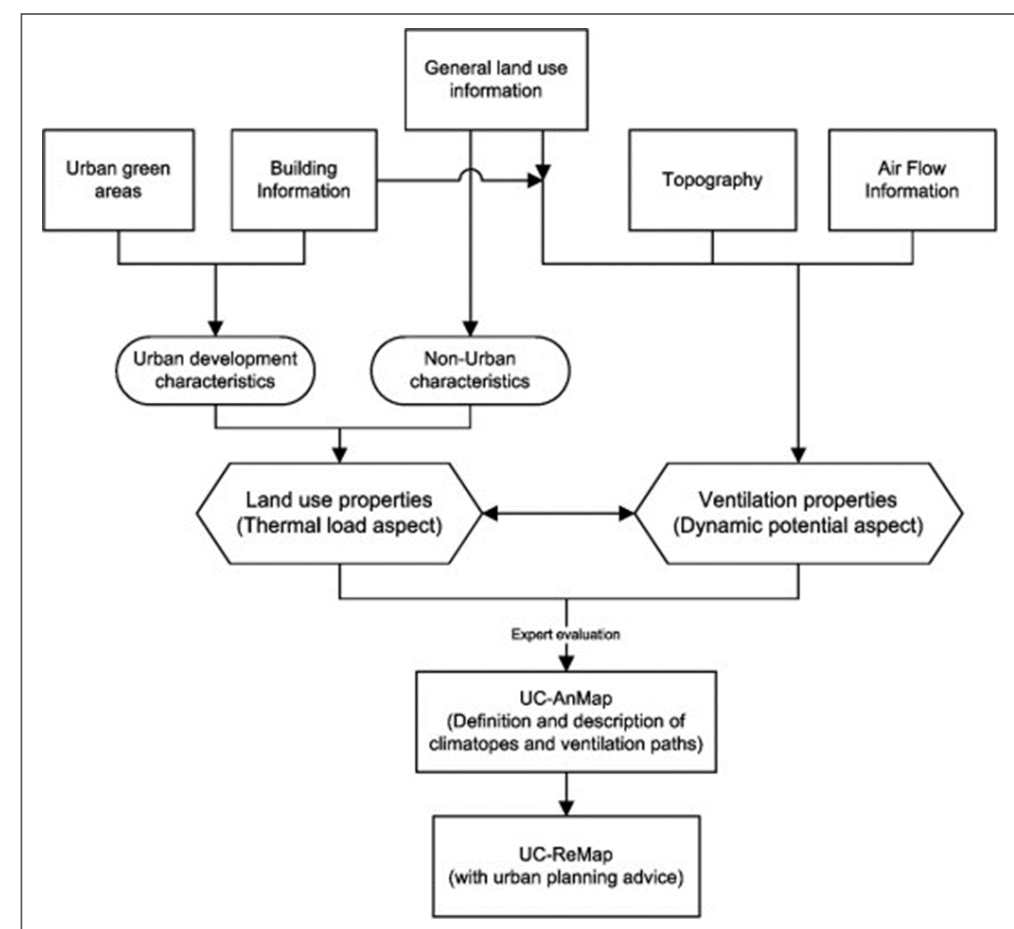

Fuente: tomado de Acero, J. A., Arrizabalaga, J., Kupski, S., \& Katzschner, L. (2013). 


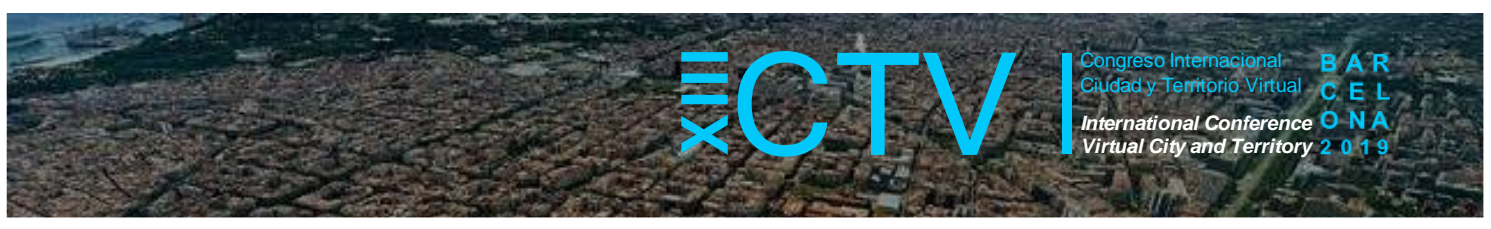

Se realiza un ejercicio de aproximación preliminar a los Local Climate Zones de nuestro SU, basado en los Climatopes que tienen menos categorías. Este es un dibujo hecho a mano del mapa para poder identificar con las distintas capas del Corine Land Cover así como con las calificaciones del MUC, buscando así la mejor manera de conseguir las distintas categorías de Local Climate Zones.

Figura 20. Ejercicio de identificación de LCZ en base a diferentes fuentes

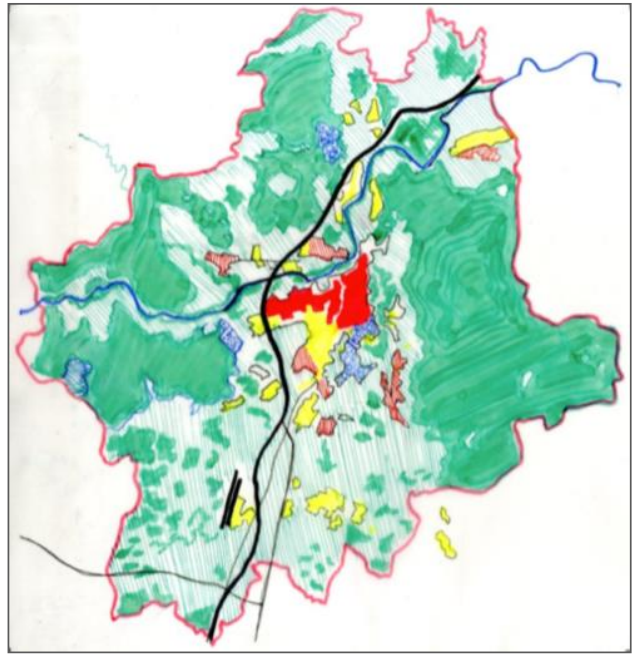

Fuente: Elaboración propia

\subsection{Definición de las LCZ}

Las Local Climate Zones fueron determinadas a partir del sistema de clasificación propuesto por Stewart y Oke (2012), que básicamente se subdividen en dos grandes grupos los Urbanos, que son numéricos (del LCZ 1 al LCZ 10) y por letras (del LCZ A al LCZ G). Se usan combinaciones entre estas también, pudiendo tener LCZ alfanuméricas. Sin embargo, en este estudio no se han considerado las mismas.

Figura 21. Local Climate Zones resultantes

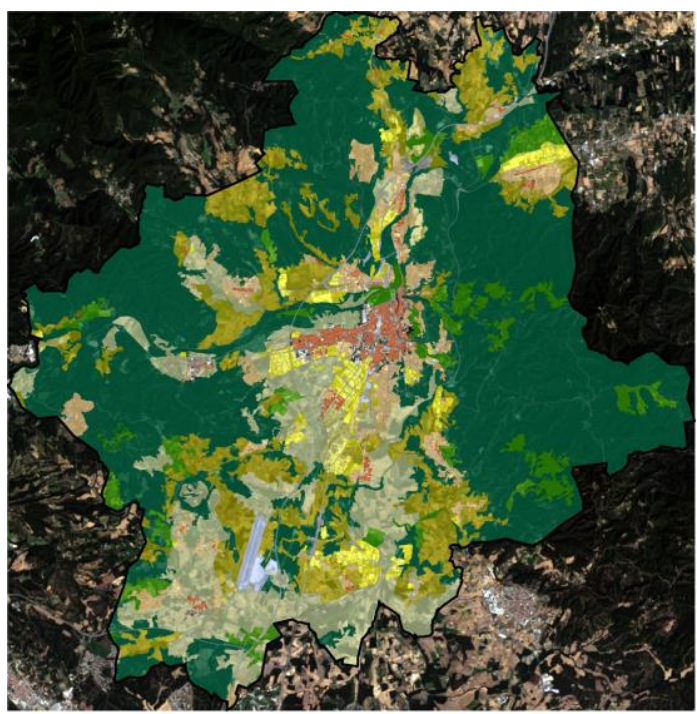

LCZ 2 - Compact midrise

LCZ 3 - Compact low-rise LCZ 5 - Open midrise

LCZ 8 - Large low-rise

LCZ 10 - Heavy industry

LCZ A - Dense Trees LCZ B - Scattered trees LCZ C - Bush, scrub LCZ D - Low plants LCZ E - Bare rock or paved LCZ F - Bare soil or sand

Fuente: CORINE Land Cover. 


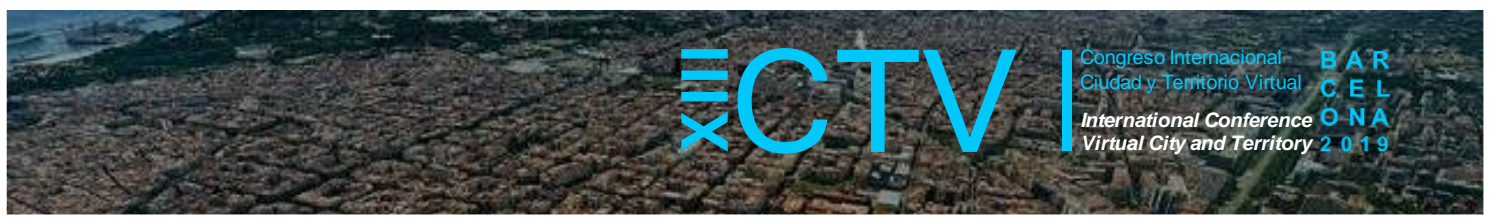

Figura 22. Detalle de resultados
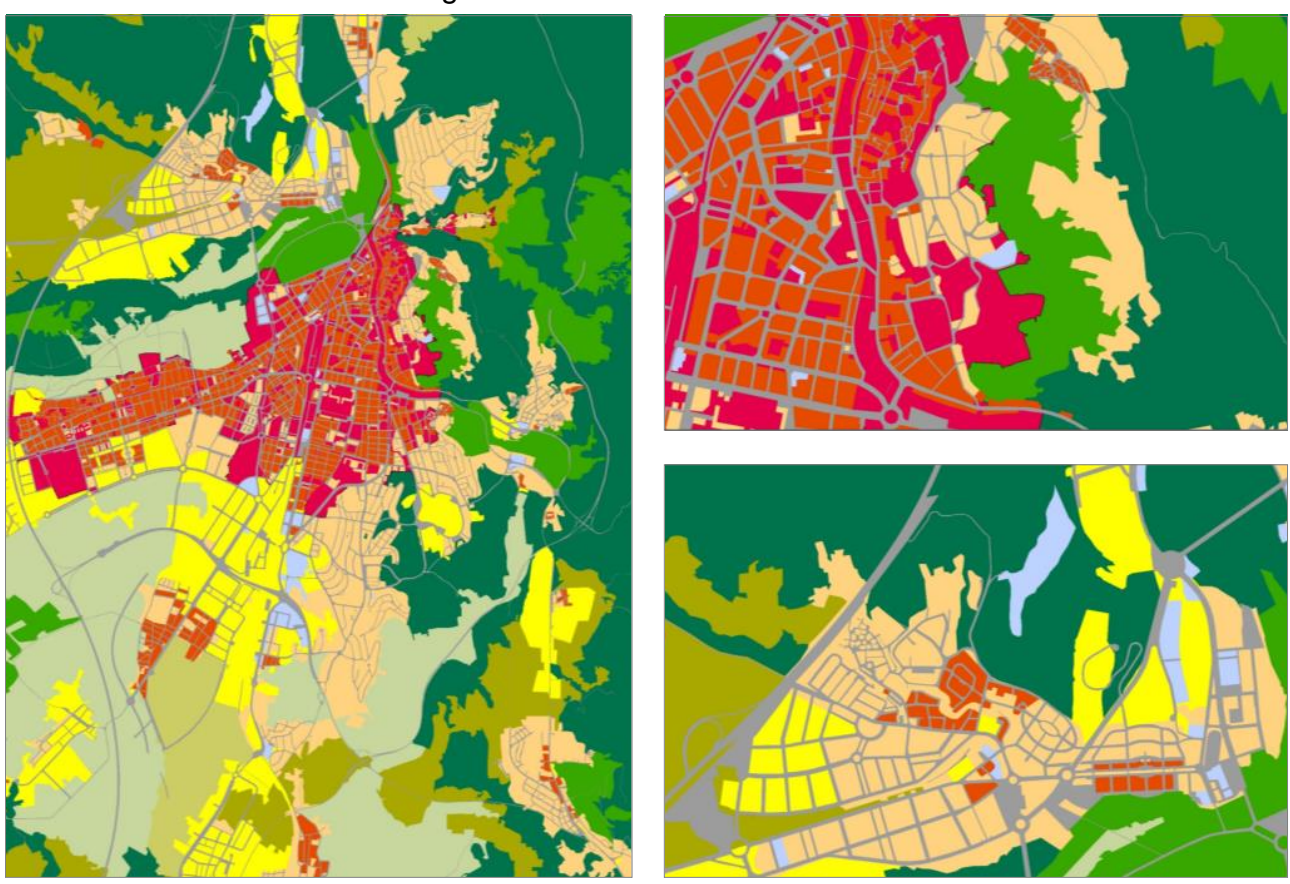

Fuente: Elaboración propia.

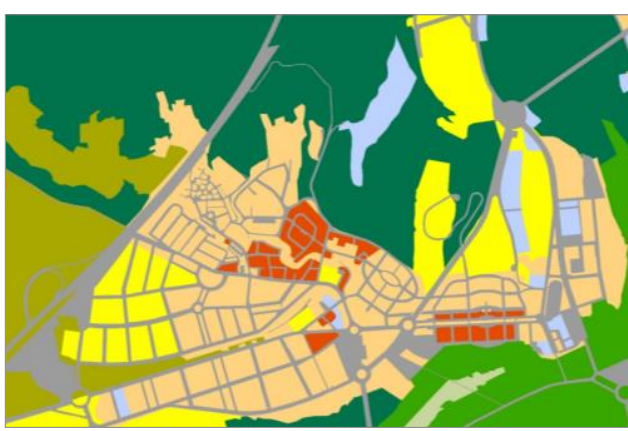

Tabla 11. Tablas estadísticas de Local Climate Zones

\begin{tabular}{|c|c|c|c|c|c|c|c|c|c|c|c|c|}
\hline & 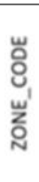 & 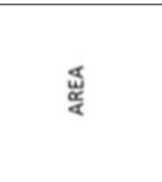 & 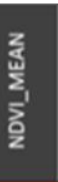 & $\frac{z}{\frac{z}{z}}$ & 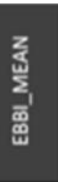 & $\begin{array}{l}5 \\
\sum_{1}^{8} \\
5\end{array}$ & 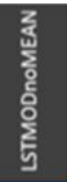 & 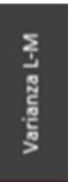 & $\begin{array}{l}\frac{w}{\Sigma} \\
w^{\prime} \\
\frac{\sigma}{z} \\
\frac{\partial}{z}\end{array}$ & 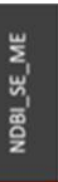 & 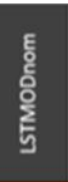 & 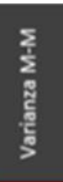 \\
\hline LCZ 8 - Large low-rise & 8 & $2.239 .200,00$ & 0,28 & 0,02 & 0,19 & 38.51 & 17,81 & 20,70 & 0,05 & 0,12 & 35,58 & 17,77 \\
\hline ICZ 10 - Heavy industry & 10 & $7.665 .300,00$ & 0,27 & $-0,02$ & 0,18 & 38,15 & 18,59 & 19,56 & 0,05 & 0,14 & 34,61 & 16,02 \\
\hline LCZ2 - Compact miderise . & 3 & $2.874 .600,00$ & 0,26 & 0,04 & 0,49 & 37,74 & 19,69 & 18,05 & 0,03 & 0,12 & 35,44 & 15,75 \\
\hline LCZ1 - Compact highrise. & 2 & $1.566 .900,00$ & 0,31 & 0,05 & 0,50 & 37,20 & 19,58 & 17,62 & 0,06 & 0,15 & 35,46 & 15,87 \\
\hline LCZ E - Bare rock or paved & E & $8.209 .800,00$ & 0,32 & $-0,05$ & 0,53 & 36,28 & 18,05 & 18,23 & 0,04 & 0,16 & 33,65 & 15,60 \\
\hline LCZE - Bare rock or paved & $E^{*}$ & $657.000,00$ & 0,32 & $-0,05$ & 0,50 & 36,22 & 18,48 & 17,74 & 0,06 & 0,15 & 34,41 & 15,93 \\
\hline ICZ 5 - Open midrise & 5 & $11.002 .500,00$ & 0,39 & $-0,06$ & 0,64 & 35,73 & 18,75 & 16,97 & 0,08 & 0,20 & 34,08 & 15,33 \\
\hline ICZ C - Bush, scrub & c & $44.715 .600,00$ & 0,40 & $-0,03$ & 0,34 & 35,59 & 17,92 & 17,67 & 0,08 & 0,19 & 33,81 & 15,90 \\
\hline LCZ D - Low plants & D & $37.745 .100,00$ & 0,45 & - 0,09 & 1,02 & 35,25 & 17,67 & 17,58 & 0,08 & 0,22 & 34,37 & 16,70 \\
\hline LCZ F-Bare soil or sand & $\mathbf{F}$ & $2.732 .400,00$ & 0,34 & $-0,06$ & 0,55 & 35,11 & 17,39 & 17,72 & 0,07 & 0,16 & 29,63 & 12,24 \\
\hline LCZ B-Scattered trees & B & $15.859 .800,00$ & 0,59 & 0,22 & 2,19 & 31,94 & 18,58 & 13,36 & 0,15 & 0,33 & 33,55 & 14,97 \\
\hline LCZ A - Dense Trees & A & $148.054 .500,00$ & 0,66 & $-0,31$ & 2,92 & 30,40 & 18,12 & 12,28 & 0,19 & 0,40 & 31,87 & 13,76 \\
\hline
\end{tabular}

Fuente: Elaboración propia

LCZ 1: Densa mezcla de edificios altos entre 6-9 pisos. Pocos o ningún árbol. Cobertura del suelo en su mayoría pavimentada. Materiales de construcción de hormigón, acero, piedra y vidrio.

LCZ 2: Mezcla densa de edificaciones de mediana altura (3-6 pisos). Pocos o ningún árbol. Cobertura del suelo en su mayoría pavimentada. Piedra, ladrillo, azulejo, y materiales de construcción de hormigón. Principalmente centro de la ciudad

LCZ 5: Arreglo abierto de edificios de media altura (3-6 pisos). Abundancia de la cobertura de la tierra permeable (plantas bajas, árboles dispersos). Materiales de construcción de hormigón, acero, piedra y vidrio. 


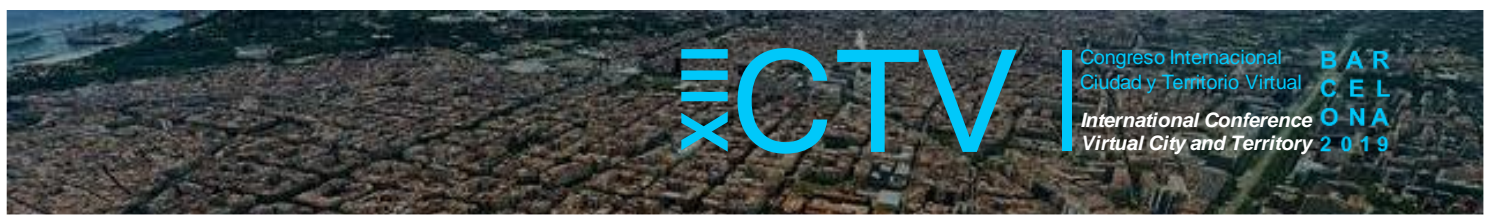

LCZ 8: Arreglo abierto de grandes edificios de baja altura (1-3 pisos). Pocos o ningún árbol. Cobertura del suelo en su mayoría pavimentada. Materiales de construcción de acero, hormigón, metal y piedra.

LCZ 10: Estructuras industriales de baja altura y media (torres, tanques, pilas) de baja o media contaminación. Pocos o ningún árbol. Cobertura del suelo en su mayoría pavimentada o empacada. Materiales de construcción de metal, acero y hormigón.

LCZ A: Paisaje muy arbolado de árboles de hoja caduca y / o siempre verde. Cobertura del suelo en su mayoría permeable (plantas bajas). La función de la zona es bosque natural, cultivo de árboles o parque urbano.

LCZ B: Paisaje ligeramente arbolado de árboles de hoja caduca y / o siempre verde. Cobertura del suelo en su mayoría permeable (plantas bajas). La función de la zona es bosque natural, cultivo de árboles o parque urbano.

LCZ C: Arreglo abierto de arbustos, arbustos y árboles cortos y leñosos. Cobertura del suelo en su mayoría permeable (suelo desnudo o arena). La función de la zona es matorral natural o agricultura.

LCZ D: Paisaje sin rastros de plantas herbáceas o herbáceas / cultivos. Pocos o ningún árbol. La función de la zona es pastizal natural, agricultura o parque urbano.

LCZ E: Paisaje sin rasgos de roca o cubierta pavimentada. Pocos o ningún árboles o plantas. La función de la zona es desierto natural (roca) o transporte urbano.

LCZ F: Paisaje sin rastros de suelo o cubierta de arena. Pocos o ningún árboles o plantas. La función de zona es desierto natural o agricultura.

\section{Conclusiones}

La determinación de la isla de calor urbano, en mayor o menor medida, dentro del sistema urbano de Girona ha sido notorio, es evidente su presencia en esta época del año y es necesaria una planificación que contemple este tipo de indicadores que sirvan para proponer las mejoras dentro del planeamiento mediante el uso de climatopes y lograr mitigar los efectos del aumento de la temperatura del territorio. Todo esto ante un evidente proceso de cambio climático que atraviesa el mundo entero, es preciso incluir estos instrumentos para dar soluciones al respecto.

Contribuciones de los autores: El primer autor ha desarrollado parte de los índices de temperatura del suelo y de temperatura atmosférica y de pequeña escala envimet, así como también elaborar la isla de calor urbana y el local climate zones, el segundo autor ha desarrollado la definición del sistema urbano y parte de los índices de temperatura del suelo y de temperatura atmosférica, el tercer autor ha desarrollado parte de isla de calor urbana y local climate zones, además de realizar el apartado de pequeña escala envimet.

Conflicto de Intereses: Los autores declaran que no hay conflicto de intereses. 


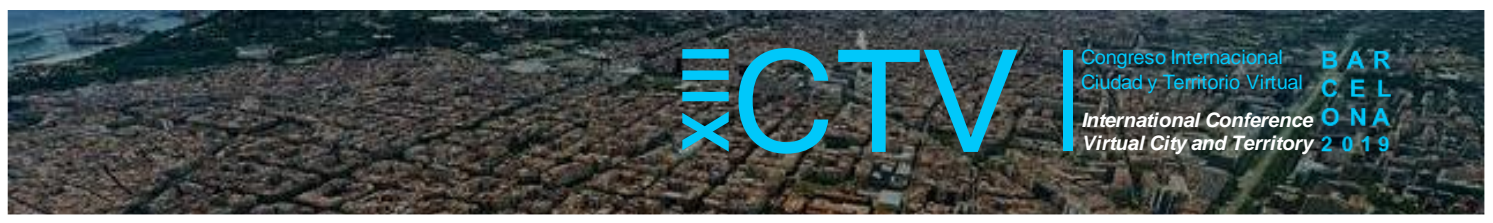

\section{Bibliografía}

ACERO, Juan A., et al. Deriving an Urban Climate Map in coastal areas with complex terrain in the Basque Country (Spain). Urban Climate, 2013, vol. 4, p. 35-60.

ACERO, Juan A., et al. Urban heat island in a coastal urban area in northern Spain. Theoretical and applied climatology, 2013, vol. 113, no 1-2, p. 137-154.

AS-SYAKUR, Abd, et al. Enhanced built-up and bareness index (EBBI) for mapping built-up and bare land in an urban area. Remote Sensing, 2012, vol. 4, no 10, p. 2957-2970.

AVDAN, Ugur; JOVANOVSKA, Gordana. Algorithm for automated mapping of land surface temperature using LANDSAT 8 satellite data. Journal of Sensors, 2016, vol. 2016.

BARSI, J. et al. The Spectral Response of the Landsat-8 operational Land Imager. remote sensing, 6 (10) 10232-10251 ISSN 2072-4292

BELLET, Carme; LLOP, Josep Maria. Ciudades intermedias: entre territorios concretos y espacios globales. Ciudad y Territorio, 2004, vol. 36, no 141-142, p. 569-581.

CASTAÑER I VIVAS, Margarida, et al. Assaig de definició de l'àrea urbana de Girona. Documents d'Anàlisi Geogràfica, 1998, núm. 33, p. 81-90, 1998.

COLL, Juli Valdunciel. Planeamiento urbanístico y forma urbana en Cataluña. El caso de la región de Girona (1979-2006). BAGE, 2014, no 65.

HUETE, A. R. Un índice de Vegetación ajustado al suelo (SAVI). Remote Sens. Enviro, 1988, vol. 25, p. 295-309.

LI, Min; XU, Lizhong; TANG, Min. An Extraction method for Water Body of Remote Sensing Image Based on Oscillatory Network. Journal of multimedia, 2011, vol. 6, no 3.

RAY, Terrill W. A FAQ on vegetation in remote sensing. California: Div. of Geological and Planetary Sciences California Institute of Technology, 1994.

OKE, T. R. Urban observations. World Meteorological Organization, IOM Report, 2004, no 81.

ROUSE JR, John W., et al. Monitoring the vernal advancement and retrogradation (green wave effect) of natural vegetation. 1973.

STEWART, Ian D.; OKE, Tim R. Local climate zones for urban temperature studies. Bulletin of the American Meteorological Society, 2012, vol. 93, no 12, p. 1879-1900.

ZHA, Yong; GAO, Jay; NI, Shaoxiang. Use of normalized difference built-up index in automatically mapping urban areas from TM imagery. International journal of remote sensing, 2003, vol. 24, no 3, p. 583-594. 